\title{
Accurate age determinations of several nearby open clusters containing magnetic Ap stars ${ }^{\star}$
}

\author{
J. Silaj ${ }^{1}$ and J. D. Landstreet ${ }^{2,1}$ \\ 1 Department of Physics and Astronomy, The University of Western Ontario, London, Ontario, N6A 3K7, Canada \\ e-mail: jsilaj@uwo.ca \\ 2 Armagh Observatory, College Hill, BT61 9DG Armagh, Northern Ireland \\ e-mail: jls@arm.ac.uk
}

Received 13 March 2013 / Accepted 9 May 2014

\begin{abstract}
Context. To study the time evolution of magnetic fields, chemical abundance peculiarities, and other characteristics of magnetic Ap and Bp stars during their main sequence lives, a sample of these stars in open clusters has been obtained, as such stars can be assumed to have the same ages as the clusters to which they belong. However, in exploring age determinations in the literature, we find a large dispersion among different age determinations, even for bright, nearby clusters.

Aims. Our aim is to obtain ages that are as accurate as possible for the seven nearby open clusters $\alpha$ Per, Coma Ber, IC 2602, NGC 2232, NGC 2451A, NGC 2516, and NGC 6475, each of which contains at least one magnetic Ap or Bp star. Simultaneously, we test the current calibrations of $T_{\mathrm{e}}$ and luminosity for the Ap/Bp star members, and identify clearly blue stragglers in the clusters studied.

Methods. We explore the possibility that isochrone fitting in the theoretical Hertzsprung-Russell diagram (i.e. $\log \left(L / L_{\odot}\right)$ vs. $\left.\log T_{\mathrm{e}}\right)$, rather than in the conventional colour-magnitude diagram, can provide more precise and accurate cluster ages, with well-defined uncertainties.

Results. Well-defined ages are found for all the clusters studied. For the nearby clusters studied, the derived ages are not very sensitive to the small uncertainties in distance, reddening, membership, metallicity, or choice of isochrones. Our age determinations are all within the range of previously determined values, but the associated uncertainties are considerably smaller than the spread in recent age determinations from the literature. Furthermore, examination of proper motions and HR diagrams confirms that the Ap stars identified in these clusters are members, and that the presently accepted temperature scale and bolometric corrections for Ap stars are approximately correct. We show that in these theoretical HR diagrams blue stragglers are particularly easy to identify. Conclusions. Constructing the theoretical HR diagram of a nearby open cluster makes possible an accurate age determination, with well defined uncertainty. This diagnostic of a cluster also provides a useful tool for studying unusual stars such as Ap stars and blue stragglers.
\end{abstract}

Key words. stars: chemically peculiar - Hertzsprung-Russell and C-M diagrams - open clusters and associations: general blue stragglers

\section{Introduction}

Open clusters are created when giant molecular clouds undergo a burst of star formation. Despite differences in the length of time required for stars of different masses to reach the zero-age main sequence (ZAMS), the entire episode of star formation is relatively short compared to the lifetime of a cluster, and hence stars in a single open cluster are considered to have approximately the same age. The members of an open cluster are also kinematically linked; they share a common space velocity (and therefore common proper motions and radial velocities) which means that membership can be strongly tested for stars that may be in nearby clusters. Furthermore, it appears that the stars of a given cluster have nearly identical initial chemical compositions. For these reasons, open clusters provide homogeneous samples of stars that make demanding tests of stellar evolution models possible, whereas such tests cannot be performed using samples of field stars because of their generally uncertain distances, bulk chemistry, and ages. Furthermore, the ages of stars on the upper

* Table 3 is available in electronic form at http://www . aanda.org main sequence (since formation and arrival on the ZAMS) are much more tightly constrained than is the case for field stars, even ones that are rather close. Thus, cluster stars make excellent laboratories for the study of evolution of stellar properties during the main sequence phase.

The possibility of determining the ages of stars in open clusters with reasonable accuracy has been exploited recently to study the evolution of magnetic field strength in intermediate mass magnetic $\mathrm{Ap}$ and $\mathrm{Bp}$ main sequence stars (Bagnulo et al. 2006; Landstreet et al. 2007, 2008). By measuring the strength of the magnetic fields in cluster Ap and Bp stars, dividing the stars into mass bins, and comparing stars of a particular mass bin but different ages (effectively using such a sequence of stars of known ages as a proxy for the evolution of a single star of that mass), it has been discovered that the magnetic fields of Ap and $\mathrm{Bp}$ stars decrease strongly during the main sequence phase of evolution. Because age determinations of field Ap stars are simply not accurate enough (Bagnulo et al. 2006), this remarkable discovery was only made possible by instrumentation advances that made it practical to detect and measure the magnetic fields of a reasonably large sample of Ap and Bp stars that are 
Table 1. Basic data for clusters studied.

\begin{tabular}{lcccccc}
\hline \hline Cluster & Parallax & DM & Distance $(\mathrm{pc})$ & $E(B-V)$ & {$[\mathrm{Fe} / \mathrm{H}]$} & $Z$ \\
\hline$\alpha$ Per & $5.63 \pm 0.09$ & $6.18 \pm 0.03$ & 177.6 & $0.09 \pm 0.02$ & $+0.02 \pm 0.03$ & 0.016 \\
Coma Ber & $11.53 \pm 0.12$ & $4.69 \pm 0.02$ & 86.7 & $0.00 \pm 0.02$ & $0.00 \pm 0.07$ & 0.015 \\
IC 2602 & $6.64 \pm 0.09$ & $5.86 \pm 0.03$ & 150.6 & $0.04 \pm 0.02$ & $-0.05 \pm 0.05$ & 0.014 \\
NGC 2232 & $2.83 \pm 0.17$ & $7.59 \pm 0.14$ & 353 & $0.02 \pm 0.02$ & $0.27 \pm 0.08$ & 0.028 \\
NGC 2451A & $5.39 \pm 0.11$ & $6.32 \pm 0.04$ & 185.5 & $0.01 \pm 0.02$ & $0.02 \pm 0.08$ & 0.016 \\
NGC 2516 & $2.90 \pm 0.08$ & $7.68 \pm 0.07$ & 345 & $0.11 \pm 0.03$ & $0.01 \pm 0.07$ & 0.016 \\
NGC 6475 & $3.31 \pm 0.13$ & $7.16 \pm 0.08$ & 302 & $0.06 \pm 0.03$ & $0.14 \pm 0.06$ & 0.021 \\
\hline
\end{tabular}

Table 2. Comparison of cluster ages $(\log t)$.

\begin{tabular}{lccccccccc}
\hline \hline Cluster & 1 & 2 & 3 & 4 & 5 & 6 & Other(s) & $\log t$ & $\sigma$ \\
\hline$\alpha$ Per & 7.55 & 7.854 & 7.71 & 7.72 & 7.3 & & $7.72^{7}, 7.7^{8}, 7.89^{9}, 7.81^{10}$ & 7.78 & 0.05 \\
Coma Ber & 8.78 & 8.652 & & & & & $8.9^{8}$ & 8.75 & 0.07 \\
IC 2602 & 7.83 & 7.507 & 7.56 & & 7.0 & 7.50 & $7.48^{11}$ & 7.56 & 0.05 \\
NGC 2232 & 7.49 & 7.727 & 7.35 & & & & $7.51^{12}$ & 7.51 & 0.05 \\
NGC 2451A & 7.76 & 7.780 & 7.56 & & 7.3 & & $7.78^{13}, 7.70^{14}$ & 7.73 & 0.07 \\
NGC 2516 & 8.08 & 8.052 & 8.03 & 8.15 & & 7.80 & $8.10^{12}, 8.20^{15}, 8.20^{16}$ & 8.08 & 0.10 \\
NGC 6475 & 8.22 & 8.475 & 8.35 & 8.35 & & 8.55 & $8.34^{17}, 8.30^{18}$ & 8.34 & 0.10 \\
\hline
\end{tabular}

References. (1) Kharchenko et al. (2005); (2) Loktin et al. (2001); (3) Mermilliod (1981); (4) Meynet et al. (1993); (5) Sanner \& Geffert (2001); (6) Tadross et al. (2002); (7) Makarov (2006); (8) Pinsonneault et al. (1998); (9) Martin et al. (2001); (10) Basri \& Martin (1999); (11) Stauffer et al. (1997); (12) Lyra et al. (2006); (13) Platais et al. (2001); (14) Carrier et al. (1999); (15) Sung et al. (2002); (16) Bonatto \& Bica (2005); (17) Sestito et al. (2003); (18) Villanova et al. (2009).

members of open clusters. For this project, where the ages of stars were obtained from those of a number of clusters, both the precision and accuracy of the cluster ages was critical. However, when we consulted the literature for cluster ages, we found quite substantial disagreements, even for nearby open clusters. Our need for accurate and unambiguous ages led us to a closer examination of possible methods for improving the determination of open cluster ages.

Age determinations of open clusters have largely been based on the fitting of theoretical isochrones to colour-magnitude diagrams (usually using $M_{V}$ or $V$ vs. $B-V$ ) from optical photometry (hereafter CMDs). Many such age determinations either do not provide an associated uncertainty, or have stated uncertainties that are quite large (often of the order of $0.3 \mathrm{dex}$, corresponding to an uncertainty factor of about 2). A number of different parameters can contribute to the final uncertainty in the derived cluster age resulting from such fitting, including uncertain distance modulus, uncertainties about cluster membership and/or binarity of individual stars, uncertain or variable reddening and extinction, uncertain cluster average chemical composition (metallicity), and uncertainties from fitting the isochrones to the available data. The complex interplay of these parameters has stimulated interesting work on maximum-probability methods of fitting cluster photometry of distant clusters to isochrones (Naylor \& Jeffries 2006; Monteiro et al. 2010); these methods not only provide optimised ages but also provide clear guidance about uncertainties of the various parameters.

In the case of young nearby clusters (within about $400 \mathrm{pc}$ ), however, most of the parameters that enter the age determination using isochrones are well determined. The distances to most nearby clusters have been determined to within a few percent from HIPPARCos parallaxes of member stars, and thus the distance moduli are known with an uncertainty of the order of $0.1 \mathrm{mag}$ or better. Cluster membership, determined from proper motions, and frequently parallaxes, for the brighter stars, is much better constrained than in distant clusters for which these data are not accurate enough to distinguish cluster stars from field stars. In most nearby clusters, the reddening is small, of the order of $0.1 \mathrm{mag}$ or less, and is known within about $0.02 \mathrm{mag}$ from the intrinsic colours of hot cluster members. Many nearby young clusters have reasonably well-known overall metallicity, and the measured metallicities do not usually depart enough from solar values to introduce age uncertainties even as large as $30 \%(0.1$ dex in $\log ($ age $))$. Thus we could expect the ages of nearby young clusters to be very accurately determined.

However, this is not the case. In Table 1, we list seven nearby open clusters that are of interest to us because of the presence in each of one or more magnetic Ap or Bp star. In this table we also list some of the principal parameters of each cluster, with our best estimate of the real uncertainties of each. In Table 2, we have collected recent determinations of the ages of these clusters. In spite of general agreement on the membership lists and the other main parameters affecting the cluster ages, the median spread in age determinations is $0.4 \mathrm{dex}$, and in the worst case is as large as 0.8 dex for IC 2602, so that the assigned ages for this cluster range from about 10 million to more than 60 million years. Since many of the extreme ages (large and small) are from relatively recent articles, in which there are no obvious errors or use of clearly obsolete methods or data, we cannot simply choose the best age determination for each cluster with confidence.

To understand this situation, we have tried to identify the aspects of age determination for these optimal clusters which have introduced the main variation from one work to another.

One possible source of age scatter could originate in the variety of computed evolution tracks and isochrones with which the observational data are compared. The available isochrone sets (e.g. Schaller et al. 1992; Bressan et al. 1993, 2012; Girardi et al. 2000; Pietrinferni et al. 2004), and consequently the ages deduced using them, differ from one another in various significant ways. The tracks are based on different detailed chemical compositions, even for nominally solar mixtures; the opacities, 
J. Silaj and J. D. Landstreet: Accurate age determinations of several nearby open clusters containing magnetic Ap stars
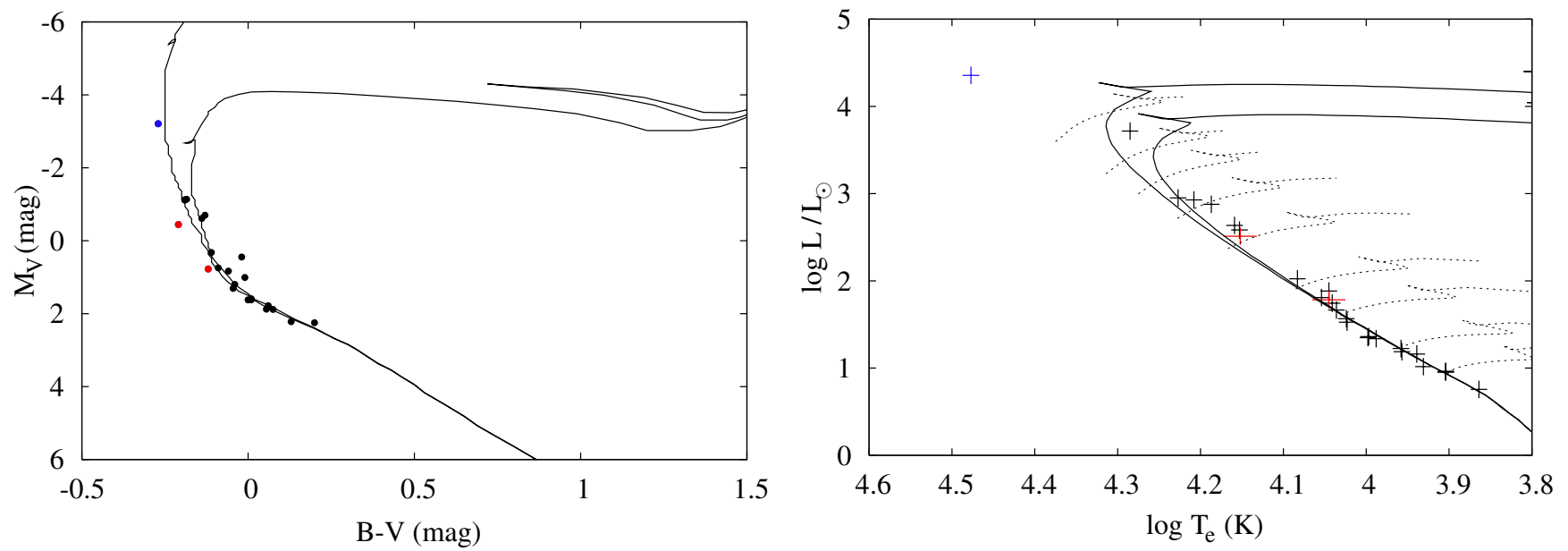

Fig. 1. Left panel: colour-magnitude diagram for IC 2602. Probable (normal) cluster members are plotted with filled black circles while Ap star members and a blue straggler are represented by filled red and blue circles, respectively. Bracketing isochrones corresponding to the limiting values found in the literature from Table $2(\log t=7.0$ (leftmost) and $\log t=7.8$ (rightmost)) are represented by solid lines. Right panel: theoretical HRD for IC 2602. Normal cluster members are plotted in black as points with error bars, Ap stars are again distinguished by red, and the blue straggler by blue, as in the left panel. Bracketing isochrones corresponding to $\log t=7.4$ (leftmost) and $\log t=7.6$ (rightmost) are represented by solid lines. Evolution tracks (from bottom right and progressing to upper left corner) for 1.7, 2.0, 2.5, 3.0, 4.0, 5.0, 6.0, 7.0, and 9.0 $M_{\odot}$ stars are represented by dotted lines. The apparent blue straggler is HD $93030=\theta$ Car.

equations of state, and nuclear reaction rates differ from one grid of models to another, and overshooting from the convective core is treated differently in different grids. The treatment of atomic diffusion varies from one calculation to another, as do the detailed numerical methods employed for the calculations. The result is that even calculations for nominally identical chemistry and age differ significantly. An example showing typical differences is found in Fig. 8 of Pietrinferni et al. (2004). Roughly speaking, the ages associated with a given isochrone using different recent computations made with similar composition and physics can differ from one another by amounts of the order of $10 \%$, or about 0.05 dex. Furthermore, the variation in the assumed metal mass fraction $Z$ appropriate to the Sun by different groups (this can range from $Z_{\odot} \sim 0.0135$ to $Z_{\odot} \sim 0.20$ ) contributes possibly as much as 20 or $25 \%$ uncertainty, or about 0.1 dex. The combination of these effects could account for at least 0.1 dex variation in the age assigned to any single cluster. However, this uncertainty, although significant, is not nearly large enough to explain the observed scatter in assigned cluster ages.

Since most of the common problems appear to contribute in only a minor way to the uncertainties in the ages of these particular clusters, we are forced to consider the actual isochrone fitting as a possible major source of uncertainty. The published cluster ages are determined mainly by comparison of the cluster member sequence in the $M_{V}$ vs. $B-V$ diagram to the best fitting isochrone. The best fitting isochrone is selected primarily by comparison of the observations to the so-called "turnoff", where the isochrone bends sharply away from the main sequence, to describe the evolution up to and past the terminal age main sequence (TAMS). In fact, we notice that this comparison is very often ambiguous, especially in the case of young clusters. This ambiguity is clearly illustrated in the left-hand panel of Fig. 1, which is a CMD showing the extrema of the ages found in the literature. We emphasise that it is not a fit to the data like the Hertzsprung-Russell diagram (HRD) depicted in the right-hand panel, but it is provided to show 1) primarily that either of the two extreme isochrones (and therefore the whole range in between, as well) plausibly fit the data, and 2) that blue stragglers cannot be easily distinguished from the normal members (which can add a significant source of error in performing the age determination). This figure shows the very different ages that result depending on whether the star to the left of the isochrone at about $M_{V} \approx-0.5$ is given high weight, and whether the very bright star at about $M_{V} \approx-3$ is considered a blue straggler or simply the most massive star in the cluster. A similar ambiguity concerning which isochrone to select is observed in the following two left panels, Figs. 2 and 3. This ambiguity occurs primarily because $B-V$ is not very sensitive to $T_{\mathrm{e}}$ above about $10000 \mathrm{~K}$.

The problem seems to be that the isochrones in the $M_{V}$ vs. $B-V$ diagram tend to be nearly vertical near the turnoff in young clusters, and this feature contributes a quite significant uncertainty in the choice of the best fit isochrone, depending on whether one chooses to fit to an isochrone with a turnoff close to the most massive stars, or one which fits the two or three hottest stars best but extends much higher in the CMD. The combination of these factors leads to large uncertainty in the age determination (usually given as $\log t$, where $t$ is the time since formation, in years).

In contrast to the CMD, the theoretical HRD uses luminosity $\log \left(L / L_{\odot}\right)$ vs. effective temperature $\log T_{\mathrm{e}}$. In this description of the cluster HRD, both axes use stellar parameters that for bright clusters may usually be obtained from a synthesis of multiple types of available information, for example photometry in both the Strömgren $u v b y \beta$ system and the Geneva system. Consequently, the values obtained for these parameters are robustly determined, and the effects of peculiarities in the spectrum of individual stars or variations in reddening across the cluster may be handled using specialised temperature calibrations and/or bolometric corrections and individual reddening corrections.

A very useful feature of the theoretical HRD is that the turnoff of the isochrones is strongly hook-shaped at all ages. Consequently, if it is possible to identify on the upper main sequence the stars that are near the end of their main sequence evolution, the observed cluster main sequence is bracketed by only a narrow range of theoretical isochrones (see e.g. the RHS of Fig. 1). As a result, working in the theoretical HRD often 

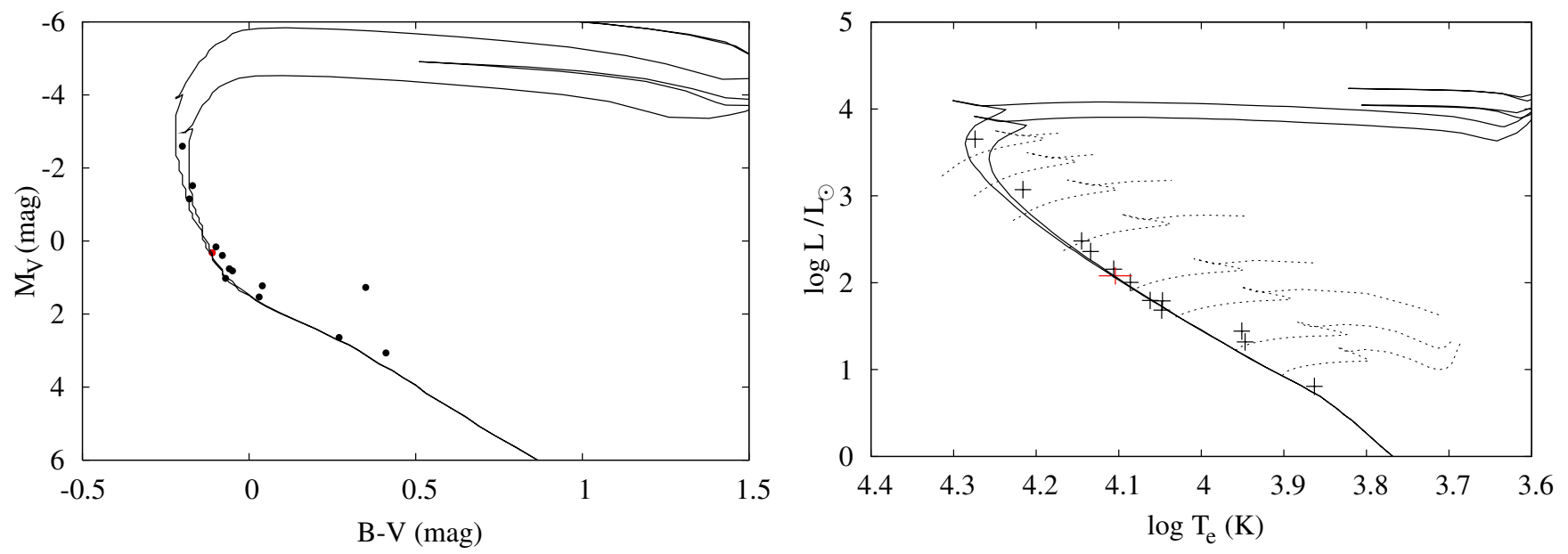

Fig. 2. Left panel: colour-magnitude diagram for NGC 2232. Symbols and colours are as in Fig. 1 with the bracketing isochrones having values of $\log t=7.4$ (leftmost) and $\log t=7.7$ (rightmost). Right panel: theoretical HRD for NGC 2232. Symbols are as in Fig. 1. Bracketing isochrones corresponding to $\log t=7.5$ (leftmost) and $\log t=7.6$ (rightmost) are shown. Evolution tracks (from bottom right and progressing to upper left corner) for 1.7, 2.0, 2.5, 3.0, 4.0, 5.0, 6.0, and 7.0 $M_{\odot}$ stars are shown.
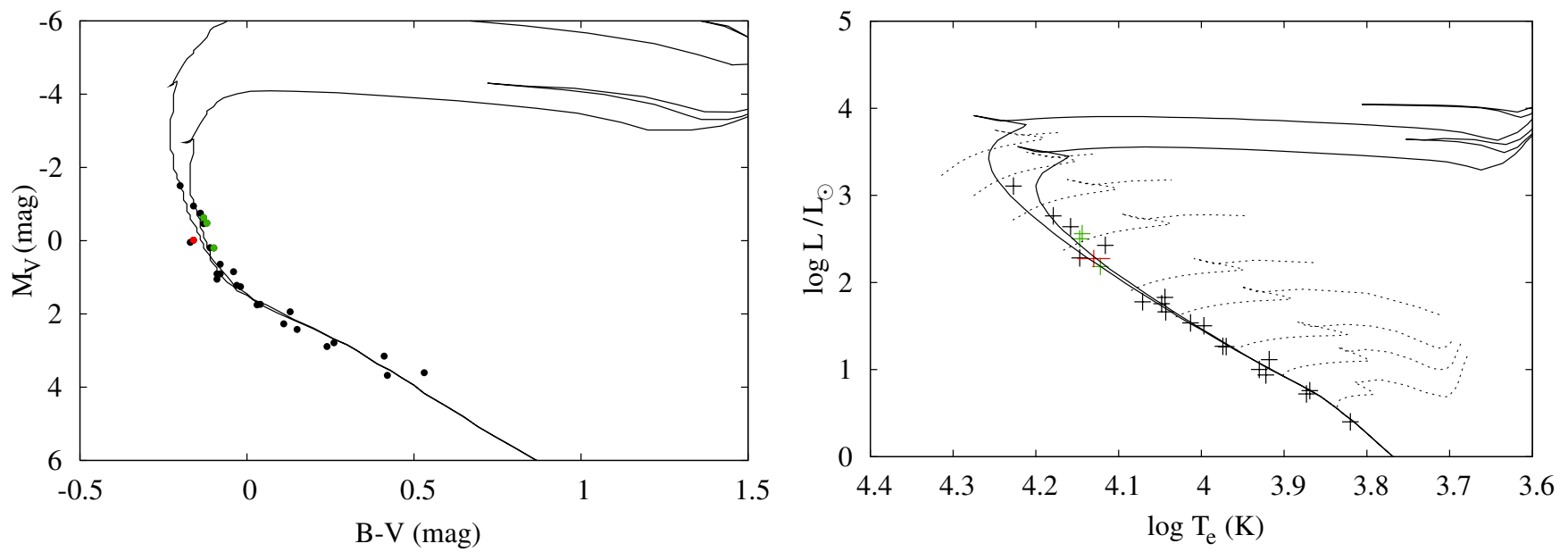

Fig. 3. Left panel: colour-magnitude diagram for NGC 2451A. Symbols are as in Fig. 1, with several cluster members that are known binaries being distinguished by filled green circles, and the bracketing isochrones having values of $\log t=7.3$ (leftmost) and $\log t=7.8$ (rightmost). Right panel: theoretical HRD for NGC 2451A. Symbols are as in Fig. 1, with the known binaries again distinguished by green. Bracketing isochrones corresponding to $\log t=7.6$ (leftmost) and $\log t=7.8$ (rightmost) are shown. Evolution tracks for 1.4, 1.7, 2.0, 2.5, 3.0, 4.0, 5.0, 6.0, and 7.0 $M_{\odot}$ stars are shown.

provides an unambiguous age for the cluster, and, equally importantly, a rather well-defined estimate of the uncertainty of the fit leading to that age. It may be possible to achieve such results while still working in the CMD if different quantities are considered - one of our referees suggests that isochrones in the $M_{B}$ vs. $U-B$ CMD would have similar properties to the isochrones in the HRD, and permit a similar unambiguous age determination for young clusters.

Our main objective in this paper, however, is to use the theoretical HRD to try to determine ages for the clusters studied that are as unambiguous and accurate as possible, and that have well-defined and relatively small uncertainties. However, the study has also produced some useful side products. First, we can confirm that the most recent proper motions, parallaxes, and HRD positions of magnetic Ap stars that are claimed to be cluster members do support cluster membership. Furthermore, the HRDs enable us to test current calibrations of effective temperature and bolometric correction used for the magnetic Ap stars, by comparing the positions of such stars to those of normal middle main sequence stars (which presumably share the same bulk structure and composition as the Ap stars) in cluster HRDs. Finally, we find that the theoretical HRD is a very suitable tool for identifying blue stragglers in young open clusters.

In the following section we discuss the sources of the data used in this study. Section 3 considers the selection of candidate members, and the determination of $T_{\mathrm{e}}$ and $\log \left(L / L_{\odot}\right)$. Section 4 outlines the general process of determining cluster ages through the examination of theoretical HRDs, while Sect. 5 discusses the ages we obtain by employing this method for the seven individual clusters studied here, and reports specific results concerning the Ap star members and possible blue stragglers. A final section reviews the conclusions reached in earlier sections.

\section{Data}

This paper investigates seven nearby open clusters: $\alpha$ Per, Coma Ber, IC 2602, NGC 2232, NGC 2451A, NGC 2516, and NGC 6475. The clusters studied are listed in Table 1 and Table 2. 
Table 1 provides a summary of basic geometric and physical parameters of each cluster, including the distance $d$, the mean parallax $\pi$, the true distance modulus DM, the mean colour excess $E(B-V)$, the mean abundance $\log \left(N_{\mathrm{Fe}} / N_{\mathrm{H}}\right)-$ $\log \left(N_{\mathrm{Fe}, \odot} / N_{\mathrm{H}, \odot}\right)=[\mathrm{Fe} / \mathrm{H}]$, and the deduced heavy element mass fraction $Z$. The references for most of these data are provided below in the discussions of individual clusters.

Cluster (logarithmic) ages derived from the literature are shown in Table 2 . Several recently published ages are provided in the columns labelled $1 \ldots 6$ and Others, with references to the sources, almost all of which are relatively recent. Our final derived ages, and their uncertainties, discussed below, are in the final two columns. This table clearly illustrates the problem of uncertainty of the ages of even these very nearby and comparatively well-studied clusters: the derived ages range over a factor of more than 2 for $\alpha$ Per, IC 2602, NGC 2451A, and NGC 2516.

All of the clusters share properties that should make accurate and precise age determinations possible. First, the open clusters studied are all within $360 \mathrm{pc}$, and are therefore relatively bright. Thus, the brighter cluster members are fairly easy to observe, even with modest instruments, and indeed many stars in each cluster are well studied and have been observed in several photometric systems. This allows us to check the reliability and consistency of the measurements and the derived fundamental stellar parameters.

The clusters in this study also all have securely determined reddening; in most cases, they are known to have little or no reddening and to lack differential reddening. (It should be noted that one cluster - NGC 2516 - does appear to have differential reddening, but the effect is small and this cluster was particularly desirable to include in the study because it contains several magnetic Ap stars.)

All of the clusters chosen for this study contain a fairly large number of middle main sequence members in addition to their magnetic star members. This is useful for defining the observed main sequence clearly, and it makes it possible to see how well the members fall onto the theoretical isochrones, and with how much scatter. Verifying that the cluster is well-behaved in this manner helps to strengthen our confidence that no large, systematic errors occur in our determinations of $\log \left(L / L_{\odot}\right)$ and $T_{\mathrm{e}}$.

As the clusters are all nearby, the individual stars in the selected open clusters have their membership and distances well constrained by astrometry. The HIPPARCOS mission (ESA 1997), and its subsequent extension to the Tycho-2 catalogue (Høg et al. 2000a,b), have been invaluable in providing high-precision proper motions and parallaxes. These datasets have allowed us to confirm many probable members, and reject other candidates whose membership was dubious, with much greater confidence than ever before.

The clusters chosen for this study contain stars for which photometric data in the Strömgren uvby and/or Geneva photometric systems are readily accessible from astronomy databases such as the SIMBAD astronomical database ${ }^{1}$, the WEBDA database for open clusters ${ }^{2}$ (Mermilliod \& Paunzen 2003), or the University of Lausanne photometric catalog ${ }^{3}$ (Mermilliod et al. 1997). Photometry in these two systems has been preferentially used because of the availability of reliable temperature calibrations and bolometric corrections for both normal and Ap stars.

\footnotetext{
http://simbad.u-strasbg.fr/simbad/sim-fid

2 http://www.univie.ac.at/webda/

http: //obswww . unige.ch/gcpd/gcpd.html
}

\section{Method}

\subsection{Candidate selection}

For each cluster, the initial selection of possible members was made using three separate catalogs: Robichon et al. (1999), Baumgardt et al. (2000), and Dias et al. (2001).

The Robichon et al. (1999) catalog is a census of cluster members of a number of nearby clusters that is based on HIPPARCos proper motions and parallaxes. Typically, the dozen or so brightest cluster members are identified. Members have been selected by an iterative process using all available data, including parallaxes, proper motions, photometry, and radial velocities. We have initially accepted all stars listed in this article as cluster members.

Baumgardt et al. (2000) have re-examined the HIPPARCos catalogue to obtain mean motions and distances of more than 300 clusters, and have used all available data to establish membership of HIPPARCos stars in these clusters. This study provides a larger sample of clusters than Robichon et al. (1999), but is limited to clusters more than about 200 pc away, so only four of the seven clusters in this study are in that catalog. However, the catalogue offers useful overlap and comparison with the others. These authors provided membership probabilities based on all available data which can be cross-referenced with other membership probability calculations. Initially all stars with non-zero membership probability were accepted from this catalogue but again, some clear non-members were later removed from the final HRDs. A very useful feature of the Baumgardt et al. (2000) catalog is that known binary stars are tagged. When constructing an HRD, it is important to be aware of binarity since (in the case of spectroscopic binaries or close visual binaries treated as single stars by photometry) the increased luminosity will shift the binary system's location upward above the main-sequence relative to single stars by as much as $0.75 \mathrm{mag}$ or $0.3 \mathrm{dex}$ in $\log \left(L / L_{\odot}\right)$, possibly leading to an error in the cluster age determination.

The catalog of open cluster members and cluster mean motions of Dias et al. (2001) is based on Tycho-2 proper motion measurements, and provides a much more extensive list of possible cluster members in the clusters of interest to us than Robichon et al. (1999). Thus the proposed list of members of each cluster spans a much wider range in temperature and luminosity. The number of fainter possible members is greatly increased because of the fainter limiting magnitude of the Tycho-2 dataset compared to the HIPPARCos data. Membership probabilities for this catalog are based essentially on proper motion data, and have been determined using the statistical method of Sanders (1971). We note that memberships based on Tycho 2 proper motions are somewhat less secure than those based on HIPPARCos motions because of the generally somewhat larger uncertainties of the former.

The clusters studied here are near enough that a substantial fraction of middle main sequence members are HIPPARCOS stars, for which the astrometric data on proper motions and parallaxes have been substantially improved by van Leeuwen (2007). The mean cluster parameters and membership issues have been extensively rediscussed on the basis of these new measurements by van Leeuwen (2009). Unfortunately, this work does not list the HIPPARCos stars that are considered by van Leeuwen to be members. However, when stars are selected from the revised HIPPARCOS catalogue of van Leeuwen (2007) with very restrictive limits on the range of parallax and proper motion, essentially the same lists of stars are found to be members of the various clusters as result from the studies above. 
For assessing membership, it is essential to keep in mind that, although the HIP stars of each cluster have astrometric data that are tightly clustered in parallax and proper motion compared to nearby field stars, the data for cluster members almost always show an intrinsic scatter, particularly in proper motion, that is two or three times larger than the uncertainties of individual astrometric data for single stars would suggest (see e.g. Figs. 10, $14,16,19$, and 23 of van Leeuwen 2009). Confirming or rejecting membership on the basis of astrometry thus requires comparison of data for individual stars with the observed spread of data values for other cluster members rather than simple measurement of the distance of a star's data set from the mean cluster values in units of standard errors.

We have initially accepted as cluster members all stars with non-zero membership probabilities in the three studies cited, but later removed any stars that are clear outliers on the HRD and that have low $(<20 \%)$ membership probabilities. The remaining stars are thus all probable cluster members. To further refine our member lists, the astrometric data for the stars retained as cluster members have been compared to the spread of astrometric data for HIP core members of each cluster, and a few significant outliers have been removed.

The stars finally retained for this study, and shown in the various figures, are listed in Table 3. This table lists the HIP number (if available) together with at least one other name, the intrinsic colour $(B-V)_{0}$ and absolute visual magnitude $M_{V}$, both corrected for the mean cluster reddening, and our adopted values of $T_{\mathrm{e}}$ and $\log \left(L / L_{\odot}\right)$, determined as described in the following section. Where one of the names provided is a cluster number designation (e.g. NGC 6475 103), the number follows the convention of of the WEBDA database. We note that these numbers are not always correctly recognised by the SIMBAD database.

The entries for each cluster in this table appear somewhat inhomogeneous. Only some of the stars in each cluster have cluster number designations, and normally the $U B V$ photometry used for the CMDs is not available for all stars. This is a consequence of the relatively recent addition to cluster membership lists of stars in the halos of the visible clusters which share the cluster proper motions and parallax. Such stars can be added to membership lists with far greater assurance with the data from the HIPPARCOS mission than was possible before. These newly identified cluster members often have HIPPARCos numbers but lack cluster numbers.

\subsection{Determination of fundamental stellar parameters $T_{e}$ and $\log \left(L / L_{\odot}\right)$}

Stellar effective temperatures were primarily determined from Geneva photometry. Geneva photometric values were given preference as they represent a homogeneous set with good cluster coverage. The six Geneva colours $U, V, B 1, B 2, V 1$, and $G$ were obtained from the University of Lausanne on-line photometric catalog and input into the program CALIB, a fortran program that returns $T_{\mathrm{e}}$ and $\log g$ values based on the calibrations proposed in Künzli et al. (1997) and described there. In this regime, the reddening of each star is specified by the user for stars of cool and intermediate effective temperatures (up to about $10500 \mathrm{~K}$ ); in the case of hot stars, the reddening is internally corrected for interstellar extinction. Adopted reddening values were taken from the literature (generally, a single value was adopted for the entire cluster membership, for reasons outlined in the previous sections). The reddening value for each cluster is given in Table 1 and in its respective subsection of
Sect. 5. Several previous investigators have constructed colourcolour (e.g. $U-B$ vs. $B-V$ ) diagrams and determined reddening by de-reddening the diagrams to line up with locally calibrated main sequence stars (see e.g. Dachs \& Kabus 1989). Since reddening is most often given in terms of $E(B-V)$, but the Geneva program requires reddening in terms of $\mathrm{B} 2$ and $\mathrm{V} 1$, the conversion $E(B 2-V 1) \simeq 0.88 E(B-V)$ as used by Hauck \& North (1993) and given by Golay (1980) was applied.

Strömgren uvby photometry values were also used to determine $T_{\mathrm{e}}$ and $\log g$, and we opted to employ this method as a secondary check on our values and to obtain values for a few clusters members for which Geneva photometry did not exist. The uvby data (specifically $V, b-y, m 1, c 1$, and $\beta$ ) were taken from the SIMBAD astronomical database and analysed by the program UVBYBETA of Napiwotzki. This program is based on the calibration of Moon and others and is described in Napiwotzki et al. (1993). UVBYBETA uses an iterative method to calculate the effective temperature $\left(T_{\mathrm{e}}\right)$ and $(\log )$ gravity of each star, and automatically calculates the individual stellar reddening value $E(b-y)$. Recalling that $E(B 2-V 1) \simeq 1.15 E(b-y)$ (see Cramer 1984; Lucke 1980; and Crawford \& Mandwewala $1976)$ and using the previously given relation for $E(B 2-V 1)$ and $E(B-V), E(b-y) \simeq 0.765 E(B-V)$.

Calculating the effective temperatures from two independent systems provides a useful check. In the majority of cases where both types of photometry were available, the calculated effective temperatures agreed within 2-300 K. Since the average temperature of our stars is around $10000 \mathrm{~K}$, the dispersion between the two systems is thus of the order of $3 \%$.

As an additional check, a third source was consulted where possible. Masana et al. (2006) use visual magnitude and 2-micron all-sky survey (2MASS) infrared photometry to obtain effective temperatures of F, G, and K-type stars (hereafter referred to as 2MASS temperatures), and claim that their new method provides uncertainties on the order of $1 \%$. While we consider this to be a very optimistic error estimate, the values they obtained nevertheless provide a useful comparison. Generally, very good agreement between the 2MASS temperatures and $u v b y$ and/or Geneva temperature determinations was found.

A minor difference between $u v b y$ and Geneva photometry is that the Geneva photometry is very homogeneous, and the tabular values available from the Geneva photometry database are averaged values. In contrast, there are often several slightly different individual determinations of $u v b y$, sometimes with outliers, and it was usually not clear which values would be the best to use.

These considerations, in combination with the fact that for many stars uvby photometry was not available (this was especially true in NGC 2232 and NGC 2451A), have led us to adopt the $T_{\mathrm{e}}$ values derived from Geneva photometry for all stars for which they were available, with the other two photometric systems providing checks on the adopted values, and $T_{\mathrm{e}}$ values for the few stars without Geneva photometry.

To compute $\log \left(L / L_{\odot}\right)$ for the stars having $T_{\mathrm{e}}>5500 \mathrm{~K}$, we used the cluster distance modulus taken from van Leeuwen (2009) (with the exception of NGC 2232, as discussed below) and individual visual magnitudes in the standard distanceluminosity relation to obtain an absolute visual magnitude $M_{V}$ for each star. The effective temperature and absolute magnitude were then used to compute the bolometric magnitude $\left(M_{\mathrm{bol}}\right)$ according to Balona (1994),

$M_{\mathrm{bol}}=M_{V}-5.5647+18.9446 \theta-19.8227 \theta^{2}+6.1302 \theta^{3}$, 
where $M_{V}$ is the visual magnitude and

$\theta=\frac{5040}{T_{\mathrm{e}}}$

For the few red giants in the clusters we obtained $T_{\mathrm{e}}$ values from Evans et al. (1996), Alonso et al. (1999), and Villanova et al. (2009). For the red giants the bolometric corrections were taken from Buzzini et al. (2010).

Finally, the relation,

$\log \frac{L}{L_{\odot}}=\frac{M_{\text {bol } \odot}-M_{\text {bol }}}{2.5}$

(where $M_{\text {bol } \odot}=4.75$ ) was used to calculate the desired luminosity parameter. For each cluster, the data sets of individual stellar fundamental parameters are plotted together with theoretical evolution tracks for stars of various masses and theoretical isochrones for $Z=0.02$, taken from Bressan et al. (1993) and Bertelli et al. (1994).

As one of the goals of this project has been to test the current calibrations of $T_{\mathrm{e}}$ and bolometric corrections for magnetic Ap stars by placing these stars in the same theoretical HRDs as normal stars in each cluster, the fundamental parameters of the apparent Ap cluster members were obtained essentially as described by Landstreet et al. (2007). Briefly, $T_{\mathrm{e}}$ values obtained from the same codes described above are corrected as proposed by Hauck \& Künzli (1996) for Geneva photometry, and as described by Stępień \& Dominiczak (1989) for Strömgren photometry. Physically, these corrections compensate approximately for the deficiency of UV flux in the energy distributions of magnetic Ap stars compared to the UV flux present in normal stars having the same Paschen continuum slope, which leads to an overestimate of $T_{\mathrm{e}}$ for a magnetic Ap star when this is estimated using the Paschen continuum. Since the flux contribution of the UV is small for late A stars and becomes more important as $T_{\mathrm{e}}$ rises, the corrections are small around 7000 or $8000 \mathrm{~K}$ and increase with $T_{\mathrm{e}}$. In contrast to our choice for normal stars, when the (corrected) values of $T_{\mathrm{e}}$ from the two types of photometry were not very different, the values were averaged and rounded.

Similarly, a slightly modified bolometric correction has been obtained for magnetic Ap stars by Landstreet et al. (2007), and this has been used for the magnetic stars to derive the luminosities.

We have included in our CMD and HRD plots all cluster members from Table 3 for which the necessary data were available. However, because the photometric data available for various cluster members is inhomogeneous, the CMD for a cluster may contain a few stars not plotted in the HRD, and vice versa. Such stars have blanks in appropriate columns in Table 3.

\section{Age determination using theoretical HRDs}

As discussed above, different treatments of the physics of the stellar models that are used to derive theoretical isochrones and ages lead to mildly different deduced ages associated with a particular isochrone. This uncertainty cannot be resolved at present. Although they incorporate the latest ideas and data, even the most recent evolution models probably still are significantly different from the structure of real stars. We estimate from comparisons of different models that the current uncertainty in the absolute scale of ages derived from models, for stars less than about $10^{9} \mathrm{yr}$ old, is probably still of the order of $0.1 \mathrm{dex}$ in $\log t$. There may be a small additional uncertainty associated with effects of rapid rotation in some stars; on the other hand, as we shall see below, possible magnetic fields seem to have little effect on the place of a star in the HRD.

However, the relative age scale uncertainty between different clusters fit to a single set of isochrones should be rather smaller than this value, as the remaining error terms in the model computations probably displace all evolution models of similar global structure (e.g. middle main sequence stars) by similar amounts. This residual relative uncertainty is probably of the order of 0.05 dex or less. Thus, it is not particularly important which set of recent isochrones we use. We have made use of several sets of evolution models and isochrones described by (Girardi et al. 2000), for metal mass fractions $Z=0.008,0.02$, and 0.05 , and our derived cluster ages are thus on the scale of this data set.

Our fits do not include an independent determination of reddening as determined from individual B stars or colourcolour diagrams, or of distance modulus as determined from HIPPARCos parallaxes (except for NGC 2232 as described below), because even without taking these as free parameters we generally have very good fits of the isochrones to the stars near the ZAMS in the HRD. Our essential single free parameter is the cluster age. The clusters studied here have fairly sparse upper main sequence membership, and so we are usually obliged to focus on one or a few stars at the upper end of the HRD. Our strategy is (1) to use the general shape of the isochrones near the best fits to identify any high-mass blue stragglers; (2) to check the most luminous stars for reports of binarity; (3) to pass isochrones around the most luminous apparently normal and single star or stars, determining the uncertainty of the fit using the isochrones that are just a little too young or too old to intersect the error boxes of the most luminous stars; and (4) to check the bracketing isochrones for general consistency with the rest of the stars on the nearby main sequence.

We typically find that this fitting procedure allows us to define a unique cluster age with an uncertainty that is at most about 0.1 dex. This is, of course, an age which is based on the assumption that the models underlying the isochrones have the same composition as the cluster stars. In fact, the clusters under study appear to have slight composition differences. To account for this, we use the reported abundances of Fe to estimate the metal mass fraction $Z$ of each cluster. Then, by comparison with a set of isochrones computed for different $Z$ values, we approximately correct the ages determined from a single set of isochrones.

All of the clusters studied here have had determinations of iron abundance $[\mathrm{Fe} / \mathrm{H}]$. The reported values (discussed for individual clusters below) range from about -0.05 to +0.32 dex, thus covering a range of about a factor of two in iron abundance. We assume that, to sufficient accuracy, the iron abundance is a proxy for overall value of $Z$, and that the two quantities vary proportionally to one another. According to Bressan et al. (2012), a reasonable choice of the solar value of $Z_{\odot}$ is 0.0152 , corresponding to an iron abundance of $\log N_{\mathrm{Fe}} / N_{\mathrm{H}}=7.52-12.00=-4.48$. We then use the reported iron abundances $[\mathrm{Fe} / \mathrm{H}]$ to determine cluster $Z$ values by adding or subtracting the (logarithmic) value of $[\mathrm{Fe} / \mathrm{H}]$ to $\log Z_{\odot}$.

From experiments we have carried out fitting a single cluster to isochrones as discussed above, we find that when we fit a given cluster, such as $\alpha$ Per, in the HRD with isochrones computed for different values of $Z$, the deduced cluster age changes. Roughly, the change in deduced age with respect to change in $Z$ relative to isochrones with $Z$ value near 0.02 satisfies $\mathrm{d} \log t / \mathrm{d} \log Z \approx-0.25$. Accordingly, we have determined 
a nominal age for each of the clusters in our study by fitting with isochrones computed for $Z=0.02$, and then corrected this age for each cluster by an amount deduced from the ratio of the cluster $Z$ to 0.02 . The largest $\log$ (age) corrections found are about 0.04 , or ten percent, with an uncertainty of less than 0.02 in general. Thus with the age corrections, the cluster to cluster variations of composition contribute only an unimportant amount to the total cluster age uncertainty.

Our final age uncertainty is generally about $\pm 0.1 \mathrm{dex}$. This uncertainty is basically the uncertainty in the relative ages of the clusters studied; it does not include the overall age scale uncertainty due to mathematical and physical approximations made in stellar modelling, which we have also estimated to be of the order of 0.1 dex. Thus, apart from the modestly uncertain overall scale of isochrone based ages, we believe that we are able to determine cluster ages to within about $\pm 30 \%$. For the clusters studied here, this result represents an important improvement in precision and accuracy of the isochrone ages, especially compared to the current spread of a factor of 2.5 ( 0.4 dex) in either direction (from 10 to $65 \mathrm{Myr}$ ) for the age of IC 2602.

\section{HRDs of individual clusters}

\subsection{IC $2602=$ Melotte 102}

This is a young nearby stellar group embedded in the Sco-Cen $\mathrm{OB}$ association, which is fairly distinctive on the sky, in proper motion, and in parallax (Kharchenko et al. 2005). It is at a distance of $d=150.6 \pm 2.0 \mathrm{pc}$ (van Leeuwen 2009), and has $E(B-V)=0.04$ (Robichon et al. 1999; Randich 2001). Previous age determinations for this cluster vary widely, from $\log t=7.0$ to 7.83 , a factor of about 7 from lowest to highest (Table 2).

The assigned ages depend strongly on whether the brightest star, $\theta$ Car $=$ HD 93030, is considered as a member, and whether it is considered a star with a normal evolutionary history or a blue straggler. $\theta$ Car is located in the central region of IC 2602. The stellar parallax is consistent with the cluster parallax at the $2 \sigma$ level, while the proper motions differ from mean cluster proper motions by about 1 mas in each coordinate, typical of the spread in proper motions of members of a nearby cluster (e.g. van Leeuwen 2009, Fig. 14). The mean stellar and cluster radial velocities, 20.2 and about $19 \mathrm{~km} \mathrm{~s}^{-1}$, are very similar (van Leeuwen 2007; Hubrig et al. 2008). It thus appears very probable that $\theta$ Car is actually a cluster member. $\theta$ Car is a chemically peculiar star, with $\mathrm{C}$ underabundant and $\mathrm{N}$ overabundant by about 1 dex each with respect to solar abundances. It is also an SB1 system with a period of $2.2 \mathrm{~d}$ and a velocity semiamplitude of $19 \mathrm{~km} \mathrm{~s}^{-1}$ in which the secondary contributes negligibly to the total light (Lloyd et al. 1995; Hubrig et al. 2008).

While the membership of $\theta$ Car seems probable, it seems unlikely from our HRD that it is a normal member: there is too large a gap between it and the next brightest member, the Be star $\mathrm{p}$ Car $=$ HD 91465. An isochrone (of $\log t \approx 6.6)$ passing through $\theta$ Car misses the second brightest star by a large margin and also falls significantly below the next five stars (see Fig. 1). If $\theta$ Car is a cluster member, then it must have had a very abnormal evolutionary history. It has often been argued that both the unusual place in the HRD, and the chemical anomalies, are the result of mass transfer from the now invisible secondary, so that $\theta$ Car is a blue straggler (e.g. Ahumada \& Lapasset 2007; Hubrig et al. 2008). Thus we disregard $\theta$ Car when we determine the age of IC 2602.

In this cluster the brightest normal member, HD 91465, which appears somewhat evolved, is bracketed by $\log t=7.4$ and $\log t=7.6$ isochrones. The cooler members all lie close to these two theoretical isochrones. Some of the B stars in the HRD scatter slightly above the isochrones, but most of them are less than $2 \sigma$ above them. One or more stars might be unidentified doubles. Using the constraint provided by the assumption that the second brightest cluster member has undergone normal single star evolution, and so should lie on an isochrone, we find the best fit log age of this cluster to be $7.52 \pm 0.05$.

We next consider the various possible additional uncertainties of this age. The reddening is only about $0.04 \mathrm{mag}$, and is uncertain by at most about $0.02 \mathrm{mag}$. The distance is known from HIPPARCos parallaxes with an uncertainty of only about 3\% (van Leeuwen 2009), and so the true distance modulus is uncertain by only about 0.03 mag. These uncertainties are included in the uncertainties of the positions of individual stars in the HRD; they mainly affect the vertical position of the cluster main sequence. The general correctness of individual star parameters and of their uncertainties is supported by the excellent fit of the lower main sequence to the computed isochrone in Fig. 1. These errors introduce no further uncertainty in the age determination beyond the fitting uncertainty.

The main remaining uncertainty is the effect of chemical composition. The iron abundance of IC 2602 is $-0.05 \pm 0.05$ (Randich et al. 2001), corresponding according to our discussion above to $Z=0.014$. This leads to an increase in the deduced $\log ($ age $)$ of +0.04 dex, which changes the age by less than its fitting uncertainty, with an uncertainty which is no more than half of the actual correction. We conclude that the dominant uncertainty is still the fitting uncertainty, and the age of IC 2602 (after correction for composition) is $7.56 \pm 0.05 \mathrm{dex}$ or $36 \pm 4$ Myr. This age and its estimated error are on the scale of the adopted isochrones, and the uncertainty is primarily the uncertainty relative to ages of other clusters determined on the same isochrone scale. If we include our roughly estimated scale uncertainty due to approximations inherent in the modelling process, the absolute age uncertainty is probably not much larger than about \pm 0.1 dex, or $\pm 20 \%$.

In Table 2 we list the ages determined here, including the abundance corrections, and with uncertainties relative to our adopted isochrone scale.

Two Ap stars are believed to be members of this cluster, HD 92385 (B9 Si) and HD 92664 (B9p Si). The proper motions and parallaxes of these two stars are within the cloud of data points in $\mu-\pi$ space, so we confirm the assumed cluster membership. These two stars are both directly detected to have magnetic fields (Bohlender et al. 1993; Bagnulo et al. 2006; Landstreet et al. 2007). A simple way to characterize the field strengths of the magnetic stars is by using the RMS value of the measurements of the mean longitudinal field $\left\langle B_{z}\right\rangle$. For the two Bp stars the RMS fields are of the order of $400 \mathrm{G}$ and $800 \mathrm{G}$, respectively. The rotation periods are, respectively, 0.55 and $1.67 \mathrm{~d}$. Their positions in the HRD, derived as discussed above, are consistent with the normal cluster stars and with the adopted isochrone.

\subsection{NGC 2232}

This cluster, identified by a few bright, stars and tight clustering in proper motion space (Kharchenko et al. 2005), is located at a distance of $d=353 \pm 22 \mathrm{pc}$, with $E(B-V)=0.02$ (van Leeuwen 2009; Robichon et al. 1999). For this cluster, almost no uvby photometry was available, so the effective temperatures computed from Geneva photometry could not be checked. Using the cluster distance proposed by van Leeuwen, all the stars 
J. Silaj and J. D. Landstreet: Accurate age determinations of several nearby open clusters containing magnetic Ap stars
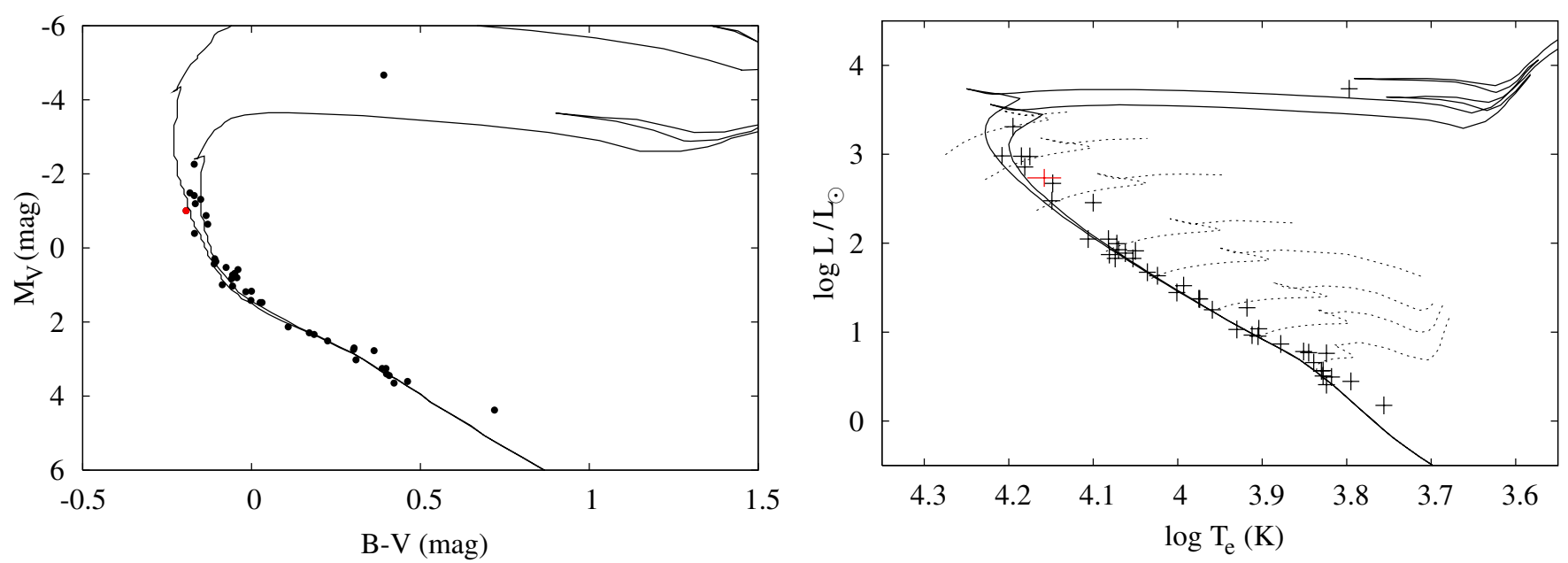

Fig. 4. Left panel: colour-magnitude diagram for $\alpha$ Per. Symbols are as in Fig. 1 with the bracketing isochrones having values of log $t=7.3$ (leftmost) and $\log t=7.9$ (rightmost). Right panel: theoretical HRD for $\alpha$ Per. Symbols are as in Fig. 1. Bracketing isochrones corresponding to $\log t=7.7$ (leftmost) and $\log t=7.8$ (rightmost) are shown. Evolution tracks for 1.4, 1.7, 2.0, 2.5, 3.0, 4.0, 5.0, and 6.0 $M_{\odot}$ stars are shown.

lie above the theoretical isochrone. The simplest assumption is that the cluster is actually somewhat nearer than the optimal value derived from the re-reduced HIPPARCos parallaxes. We have plotted the stars assuming that the actual distance modulus is $1 \sigma$ smaller than proposed by van Leeuwen, i.e. 7.59 rather than 7.73 mag. With this choice the stars generally lie close to the isochrone.

The iron abundance of NGC 2232 is reported to be $0.27 \pm$ 0.08 dex above the solar value by Monroe $\&$ Pilachowski (2010), leading to an inferred metallicity of $Z \approx 0.028$. We note that using isochrones calculated for larger metallicity than the nominal value of $Z=0.02$ would raise the position of the computed main sequence in the HRD, but not by enough explain the discrepant position of the main sequence with van Leeuwen's parallax value. We still need to adjust the distance downward to fit the cluster main sequence.

Ages estimated for this cluster recently range from 7.35 to 7.73 (Table 2). The brightest member, HD $45546=$ HIP 30772, is bracketed by $\log t=7.5$ and $\log t=7.6$ isochrones, leading to a nominal age, using the $Z=0.02$ isochrones, of $\log t$ for this cluster of $7.55 \pm 0.05$. Applying a metallicity correction of -0.04 dex, our final log age is $7.51 \pm$ 0.05 , or about $32 \pm 4$ Myr.

No blue stragglers have been identified in this cluster (Ahumada \& Lapasset 2007), and none appear in our HRD.

The Ap star in this cluster, HD 45583 (B9 Si), has astrometric data which are fully consistent with membership in the cluster. The star has a very large field, with an RMS value of about $2700 \mathrm{G}$, which varies on a period of $1.177 \mathrm{~d}$ (Bagnulo et al. 2006; Kudryavtsev et al. 2006; Landstreet et al. 2007; Semenko et al. 2008). Its position in the HRD is normal.

\subsection{NGC 2451A = Puppis Moving Group}

NGC 2451A is an indistinct nearby group of stars with welldefined proper motion and parallax (Kharchenko et al. 2005; van Leeuwen 2009). The cluster proper motion region is well separated from the proper motion cloud of nearby field stars, so membership is fairly unambiguous. The distance of this cluster is $d=185.5 \pm 3.7 \mathrm{pc}$ (van Leeuwen 2009), and the colour excess is $E(B-V)=0.01$ (Carrier et al. 1999). The cluster photometry is generally well-behaved, and good agreement between the uvby and Geneva temperatures was found wherever both kinds of photometry were available. Recent previous age determinations range from 7.3 to 7.78 (Table 2).

The iron abundance of this cluster is $[\mathrm{Fe} / \mathrm{H}]=0.02 \pm 0.08$ (Hünsch et al. 2004), leading to $Z=0.016$ and a log age correction relative to $Z=0.02$ isochrones of +0.03 dex.

The brightest member of the cluster does not seem to be very evolved, but is best fit by a $\log t=7.6$ isochrone. Several other less evolved bright members may be better modelled by the $\log t=7.8$ isochrone. We note, however, that some of the brighter members of this cluster are found to be binary stars, and hence may lie up to $0.75 \mathrm{mag}$ above the isochrone. The nominal $\log t$ for this cluster is $7.70 \pm 0.07$, which we correct to $7.73 \pm 0.07 \mathrm{dex}$, or about $54 \pm 8 \mathrm{Myr}$.

According to Ahumada \& Lapasset (2007), it has been suggested that the stars HD 61831 and HD 63465 are blue stragglers. The parallax and proper motions of HD 61831 indicate that the star is, with high probability, a member, but it lies on the isochrone of the cluster in our HRD, and is not a blue straggler. The parallax and proper motions of HD 63465 are very different from those of the cluster (van Leeuwen 2007), and this star is certainly not a member.

The one known Ap star member of the cluster, HD 63401, is an Ap Si star. It is a HIP star, and its redetermined astrometric parameters are fully consistent with cluster membership. It has a rotation period of $2.41 \mathrm{~d}$ (Hensberge et al. 1976). This star has a firmly detected magnetic field with an RMS value of about $350 \mathrm{G}$ (Bagnulo et al. 2006; Landstreet et al. 2007). The star occupies a normal place in the HRD.

\section{4. $\alpha$ Per $=$ Melotte 20}

This large, bright, and nearby cluster occupies a very distinctive place in proper motion and parallax space (Sanner \& Geffert 2001; Kharchenko et al. 2005; van Leeuwen 2009). It is at a distance of $d=177.6 \pm 2.7 \mathrm{pc}$ (van Leeuwen 2009), and has significant reddening of $E(B-V)=0.09 \pm 0.02$ (Robichon et al. 1999; Sanner \& Geffert 2001). The cluster has a wide range of stars for which photometric measurements are available, and using Geneva photometry to obtain $T_{\mathrm{e}}$ values, most members coincide very well with theoretical isochrones (see Fig. 4). Recent log age 

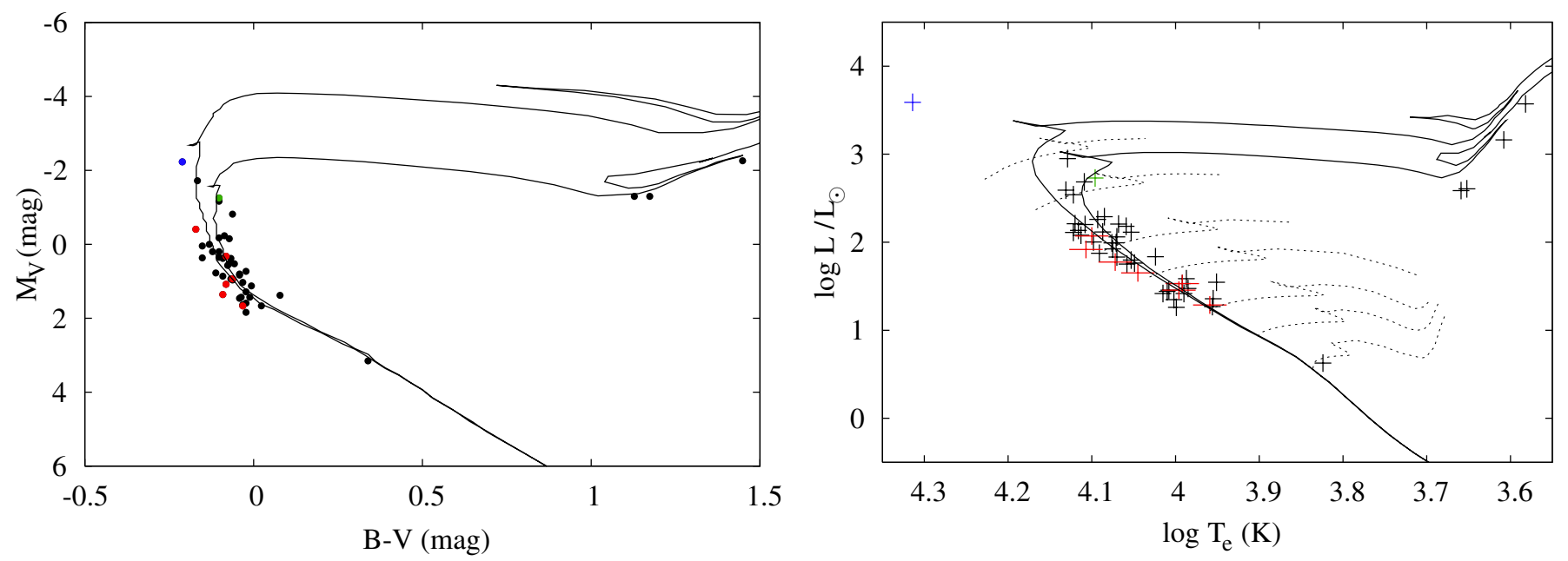

Fig. 5. Left panel: colour-magnitude diagram for NGC 2516. Symbols are as in Figs. 1 and 3 with the bracketing isochrones having values of $\log t=7.8$ (leftmost) and $\log t=8.2$ (rightmost). Right panel: theoretical HRD for NGC 2516. Symbols are as in Figs. 1 and 3. Bracketing isochrones corresponding to $\log t=7.9$ (leftmost) and $\log t=8.1$ (rightmost) are shown. Evolution tracks for 1.4, 1.7, 2.0, 2.5, 3.0, 4.0, and 5.0 $M_{\odot}$ stars are shown. The apparent blue straggler is HD 66194.

estimates range from 7.3 to 7.9 , or from about 20 to 80 million yr (Table 2).

According to Boesgaard et al. (2003), $[\mathrm{Fe} / \mathrm{H}]=+0.02 \pm$ 0.03 dex for the $\alpha$ Per cluster. This leads to an estimated cluster $Z=0.016$ and a $\log$ age correction relative to $Z=0.02$ isochrones of +0.03 .

In the theoretical HRD, the age of this cluster is most strongly constrained by the position of the brightest main sequence star in the cluster, $\psi$ Per (HD 22192), which lies close to the TAMS. van Leeuwen (2009) strongly argues that this star is indeed a member of the cluster. If the bracketing isochrones are chosen to be just a little younger and just a little older than values which just intersect the error bars of this star, we find $\log t=7.75 \pm 0.05$, which after correction becomes $7.78 \pm 0.05$, or an age of about $60 \pm 7$ Myr. The two bracketing isochrones also intersect satisfactorily the error ovals of other stars near the turnoff (except for two stars which may be unrecognised binary systems, and lie a bit above the general trend), although these other stars do not constrain the cluster age as tightly as $\psi$ Per. The bright red giant after which the cluster is named lies on the younger of the two bracketing isochrones. This cluster is another example of a group whose age can be strongly constrained by a single star near the TAMS.

According to Ahumada \& Lapasset (2007), the star $\delta$ Per $=$ HD 92928 may be a blue straggler. However, the proper motion $\mu_{\delta}$ is nearly 20 mas/a different from the motion of the cluster, so this star is clearly not a cluster member.

The one well-studied Ap star in $\alpha$ Per, HD 21699, is a B8 He weak star with strong Mn. The new HIP astrometric parameters place the star within the cloud of points of other cluster members. The star has an RMS field of the order of $600 \mathrm{G}$ and a rotation period of $2.49 \mathrm{~d}$ (Brown et al. 1985). The position of HD 21699 in the HRD agrees well with the neighbouring stars and with the theoretical isochrones.

The star HD 22401 has been suggested to be another Ap $(\mathrm{SiSrCr})$ member of the cluster. Its proper motion and parallax are consistent with membership. However, unpublished observations made by one of the authors with the ESPaDOnS spectropolarimeter at the Canada-France-Hawaii Telescope (CFHT) indicate that this star has no detectable magnetic field, and spectrum modelling shows that the star has atmospheric abundances close to solar, so it is very probably not a magnetic Ap star at all.

\subsection{NGC 2516}

NGC 2516 is a large, rich cluster with more than 300 members. Its proper motion is similar to those of nearby field stars, but the proper motions of members are confined to a small, well-defined cloud in proper motion space. The cluster seems to be a richer version of the Pleiades cluster, with a similar age. NGC 2516 is at a distance of $d=345 \pm 11 \mathrm{pc}$, and has $E(B-V)=0.112 \pm 0.024$ (Sung et al. 2002). The reddening of the cluster is mildly variable, as characterized by the uncertainty given for the $E(B-V)$ value. Differential reddening to this small degree affects temperature determinations in a relatively minor way and should be more than accounted for by the error bars associated with our $T_{\mathrm{e}}$ estimates. The large number of stars, and of Ap stars in particular, in this cluster make it a very interesting agglomeration to study, despite the variable reddening. Recent age determinations for this cluster show less scatter than are found for most of the other clusters studied here, and range from 7.8 to 8.2 (Table 2), probably because the three red giant members of the cluster can be used to strongly constrain the appropriate isochrone in the CMD.

Maiorca et al. (2011) report that $[\mathrm{Fe} / \mathrm{H}]=+0.01 \pm 0.07$, leading to $Z=0.016$ for this cluster. The corresponding correction to ages derived from $Z=0.02$ isochrones is +0.03 .

Although the uvby and Geneva temperature determinations were in good agreement for this cluster, there is an obvious scatter of the stellar data about the isochrone. It is not completely clear why this cluster exhibits what seems to be "excess" scatter; this scatter is strong above $T_{\mathrm{e}}=10000 \mathrm{~K}$, where correction for reddening is built into both temperature calibrations. Possibly the cluster has an abnormally large number of unrecognized binary systems. In addition, the membership list is less secure than for most of the other clusters studied here; as mentioned above, the proper motions of cluster members are very similar to the motions of the field stars in this region of the sky (Kharchenko et al. 2005), and the cluster is distant enough that measured parallaxes do not provide a strong discriminant against foreground or background non-members.

The majority of stars in the middle of the diagram would be best fit by the lower isochrone at $\log t=7.9$, but the brighter members near the turnoff clearly adhere more closely to the upper bracketing isochrone of $\log t=8.1$ (see Fig. 5). We adopt a 
J. Silaj and J. D. Landstreet: Accurate age determinations of several nearby open clusters containing magnetic Ap stars
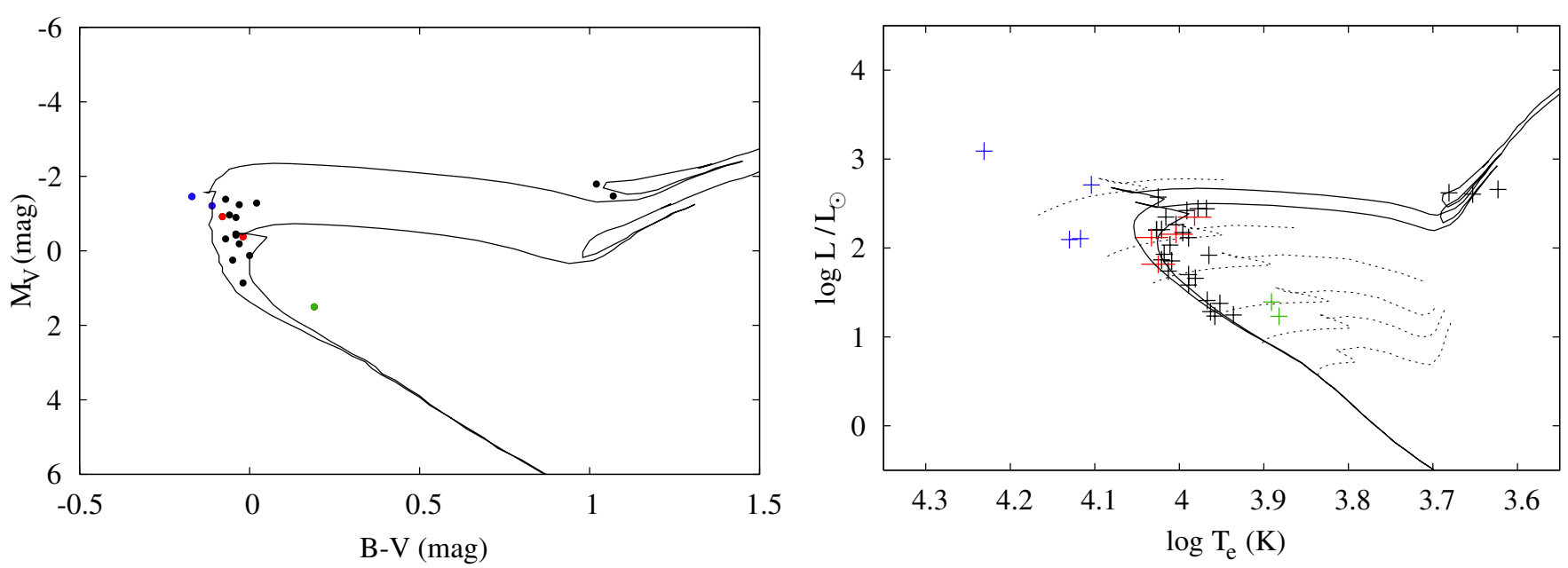

Fig. 6. Left panel: colour-magnitude diagram for NGC 6475. Symbols are as in Figs. 1 and 3 with the bracketing isochrones having values of $\log t=8.2$ (leftmost) and $\log t=8.6$ (rightmost). Right panel: theoretical HRD for NGC 6475. Symbols are as in Figs. 1 and 3 . Bracketing isochrones corresponding to $\log t=8.3$ (leftmost) and $\log t=8.4$ (rightmost) are shown. Evolution tracks for 1.4, 1.7, 2.0, 2.5, 3.0, and 4.0 $M_{\odot}$ stars are shown. The extreme blue straggler at $\log T_{\mathrm{e}}=4.23$ is $\mathrm{HD} 162374$.

corrected age of $\log t=8.08 \pm 0.1$, about $120 \pm 25$ Myr. This age is supported by the fit of the cluster's three red giants to the isochrones.

This age estimate disregards the star HD $66194=$ HIP 38994 = V374 Car, seen in the upper left corner of Fig. 5. This star has been identified as a blue straggler in the list of Ahumada \& Lapasset (2007). The proper motions and parallax of the star from the new reductions of the HIPPARCos data (van Leeuwen 2007) place it well within the cloud of proper motion points for cluster members shown in Fig. 19 of van Leeuwen (2009), and the stellar parallax is consistent (within less than $1 \sigma)$ with the cluster distance. HD 66194 is apparently a spectroscopic binary (Gonzales \& Lapasset 2000), but since the period is not known, we cannot use the stellar radial velocity to test membership. However, in the HRD the position of HD 66194 is consistent with membership, assuming that this star has had abnormal evolution. We conclude that on balance it is very likely that this star is indeed both a genuine cluster member and a blue straggler.

The cluster contains several stars which have been classified as magnetic Aps. None of these stars are HIPPARcos stars, and so none have newly revised, high-accuracy proper motions. Instead, the proper motions are obtained from the Tycho data. The astrometric properties of all these Ap stars are consistent with cluster membership, but since the uncertainties of the measured motions are of the order of 3 or 4 mas/a, membership is not nearly as secure as for the majority of Ap and Bp stars studied in this work.

Magnetic fields have been detected in four of the cluster Ap and Bp stars (Bagnulo et al. 2006; Landstreet et al. 2007). HD 66318 (A0p SrCrEu), the coolest of these four, has a huge RMS field of about $4400 \mathrm{G}$, and has been studied in detail by Bagnulo et al. (2003). The three hotter Ap stars with detected fields, HD 65712 (A0p Si 4200), HD 65987 (B9p Si), and HD 66295 (B8/9p Si), all have RMS fields in the range of 500-900 G. Rotation periods have not been established for any of these four stars with real certainty, but that of HD 66295 is probably $2.45 \mathrm{~d}$. The remaining Ap stars in NGC 2516 plotted in Fig. 5 have been surveyed for fields once or twice each, but the fields were below the threshold for detection. The magnetic
Ap stars scatter about the bracketing isochrones about as well, or as poorly, as the normal stars.

\section{6. $N G C 6475=$ Messier 7}

NGC 6475 is a sufficiently bright and obvious cluster to have attracted the attention of Charles Messier in the 18th century. It is at a distance of $d=302 \pm 10 \mathrm{pc}$ (van Leeuwen 2009), and has $E(B-V)=0.06$ (Meynet et al. 1993; Robichon et al. 1999). It should be noted that membership determinations for the cluster are hampered by the fact that the mean motions of the cluster are very close to the mean motions of field stars in the same direction, although the cluster motions are tightly bunched. Perhaps for this reason, several stars that have been thought to be probable members in previous studies appear from their positions in our HRD or from discrepant proper motions to be non-members (for example, HD 162205 = NGC 64753 lies far enough below the isochrones of Fig. 6 to be regarded as a probable nonmember).

The metallicity, according to Sestito et al. (2003) is $+0.14 \pm$ 0.06 , slightly above solar. The corresponding $Z=0.021$, and the age correction to isochrone ages from $Z=0.020$ computations is -0.01 dex.

It was found that the temperatures derived from the calibrations of Geneva $X Y$ and $p T p G$ photometry were somewhat discrepant for a few stars of this cluster that have $T_{\mathrm{e}}$ values right at the boundary between the two calibrations, at about $10000 \mathrm{~K}$. In such cases an average of the $T_{\mathrm{e}}$ values produced by the two calibrations was generally taken. Two stars that lie above and to the right of the isochrones, marked by asterisks, are known to be spectroscopic binaries, and a third star, HD 162781, that is separated from other stars inside the curve of the isochrone, may also be a binary.

Previous age determinations (Table 2) range from $\log t=$ 8.22 to 8.55 . Our best (corrected) age estimate, considering the breadth of the main sequence in our HRD, is $8.34 \pm 0.10$, or about $220 \pm 50$ Myr. This cluster is old enough that age determinations in the CMD benefit from the more hooked shape of isochrones (compared to those for young clusters) as stars evolve towards the TAMS, so that working in the theoretical 

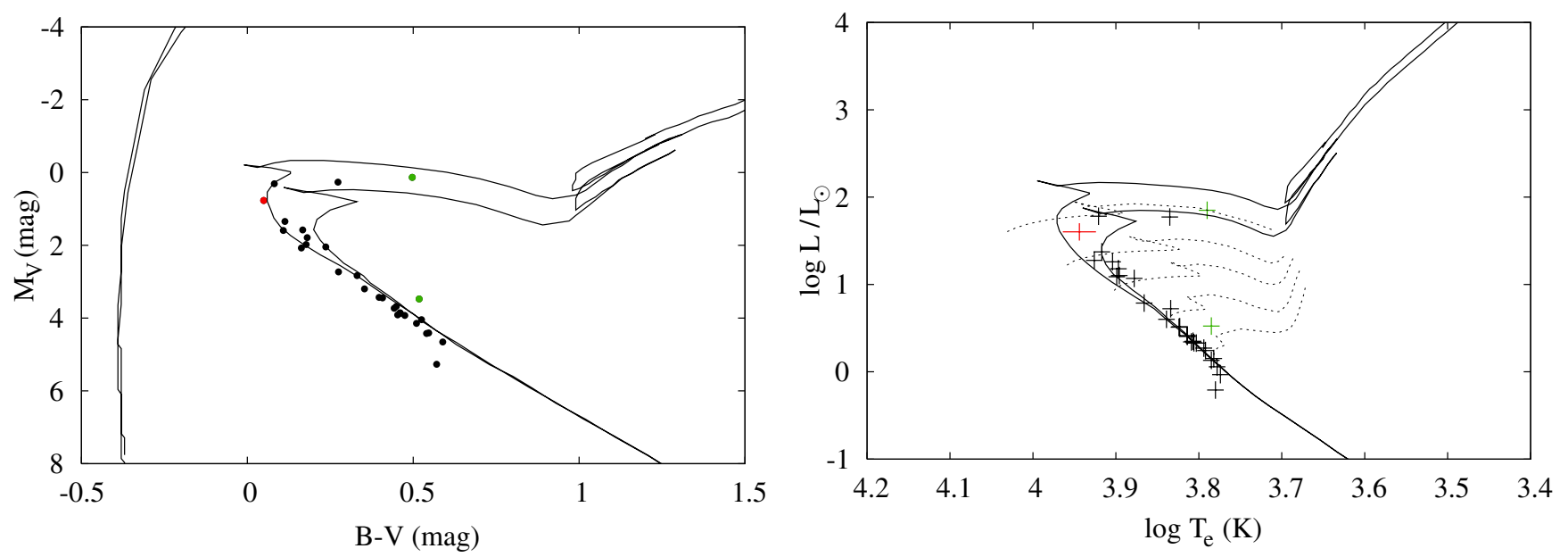

Fig. 7. Left panel: colour-magnitude diagram for Coma Ber. Symbols are as in Figs. 1 and 3 with the bracketing isochrones having values of $\log t=8.7$ (leftmost) and $\log t=8.9$ (rightmost). Right panel: theoretical HRD for Coma Ber. Symbols are as in Figs. 1 and 3 . Bracketing isochrones corresponding to $\log t=8.6$ (leftmost) and $\log t=8.8$ (rightmost) are shown. Evolution tracks for 1.1, 1.4, 1.7,2.0, and 2.5 $M_{\odot}$ stars are shown.

HRD does not increase the precision of age estimation as much as for younger clusters. In addition, red giant members help to define the age in both the CMD and the HRD.

According to the summary of Ahumada \& Lapasset (2007), two stars have previously been identified as blue stragglers: HD 162374 and HD 162586. Both stars have HIPPARCos parallaxes and motions that are consistent with membership (van Leeuwen 2007). The brighter of these stars, HD 162374, is particularly interesting. If it is indeed a member, its location in our HRD certainly identifies it as a blue straggler, similar to $\theta$ Car in IC 2602, and to HD 66194 in NGC 2516. HD 162586 may be a different kind of blue straggler. Its position in our HRD is significantly hotter than the stars that cluster about the isochrone, and near the TAMS. This star may be a mild blue straggler that is completing its delayed main sequence evolution.

Our HRD shows two other stars that appear to be blue stragglers. HD 161649 and HD 163251 both appear to be fairly secure members, but both are substantially too hot to lie on the cluster isochrones. Both appear to be still close to the ZAMS.

This cluster contains several Ap stars, among the largest number found in any of the clusters in this study. The chemical abundances of several of these stars have been studied by Folsom et al. (2007). Four Aps are plotted in Fig. 6. HD 162576 (B9.5p SiCr) and HD 162725 (A0p SiCr) are both HIP stars, with revised proper motions and parallaxes that are consistent with membership. Both have RMS fields of a few tens of $G$, firmly detected with ESPaDOnS at the CFHT. The fields of HD 162305 (B9p) and HD 162588 (Ap Cr) are so far undetected (Landstreet et al. 2008). The Tycho motions of these two stars are consistent with membership; since these particular Tycho data have error bars only a little larger than 1 mas/a, this is a fairly strong result. The small field values of Ap stars in this cluster, compared to those mentioned above for stars in younger clusters, are consistent with the general decline in field strength with age found by Landstreet et al. (2008). These stars all lie within in the general spread of normal stars about the isochrones.

\subsection{Coma Ber $=$ Melotte 111}

The cluster in Coma Berenices is a sparse group of stars at high galactic latitude. It is at a distance of $d=86.7 \pm 0.9 \mathrm{pc}$ (van Leeuwen 2009), with a colour excess of $E(B-V)=0.00$ (Robichon et al. 1999). Although the cluster mean motions lie well within the cloud of field star proper motions in this direction, the cluster has a very tight distribution compared to the field, and membership can be strongly tested.

Gebran et al. (2008) find that the Fe abundance in this cluster is essentially solar, so the value of $Z=0.015$ and the age correction is about +0.03 dex.

The cluster is the oldest cluster we investigated, and thus the main sequence has only stars later than about A2 (Fig. 7). We found that the uvby, Geneva, and 2MASS temperatures were in good agreement with one another and placed the stars on the theoretical isochrone. In this cluster, the final plot includes only stars for which Geneva photometry is available. The range of ages proposed in recent work goes from 8.65 to 8.9 ; as in NGC 6475, the advanced age of this cluster means that in the photometric CMD the isochrones have a sharp hook near the top of the main sequence and so ages are relatively precisely determined directly from CMDs. From the best fit isochrones in the HRD, our corrected adopted age is $\log t=8.75 \pm 0.07$, or about $560 \pm 90$ Myr.

Ahumada \& Lapasset (2007) report that the star HD 108662 , a magnetic Ap star, has been identified as a blue straggler. However, the proper motions of this star, as reported by van Leeuwen (2007), are completely inconsistent with membership. This star is not a member of Mel 111.

The one well-established magnetic Ap star in the cluster is HD $108945=21 \mathrm{Com}$, an A2p SrCr star with a rotation period of 2.0 days and a weak but unambiguously detected magnetic field with an RMS value of around $150 \mathrm{G}$ (Landstreet et al. 2008). The revised astrometric parameters of this star confirm that it is a cluster member. This star is currently one of the most evolved main sequence stars in the cluster. Its position on the isochrone is normal.

\section{Summary and conclusions}

This work started from the need for precise and accurate age determinations for a number of nearby clusters in order to obtain accurate relative elapsed ages since the ZAMS of magnetic 
stars in these clusters. We observed that recent measurements of the ages of these clusters, based on stellar evolution calculations and isochrone fitting, can differ by factors of 2 or 3, even though membership in the clusters is largely well-established, the distances are accurately known, and there is little reddening.

We have tried to determine the sources of the wide range of ages published for such clusters as $\alpha$ Per and IC 2602. We considered the possible effects of differences in cluster membership and cluster parameters, as well as differences between various isochrone computations and those due to differences in chemical compositions between clusters.

We were unable to find any reasonable source for the spread in reported ages except for the various ways in which the isochrones are fitted in the $\left(M_{V}\right.$ or $V$ vs. $\left.B-V\right)$ CMD. We have experimented with isochrone fitting in the theoretical HRD and we conclude that fitting cluster photometry in the HRD provides an unambiguous way to determine the ages of these particularly favourable clusters, and the uncertainties in these ages. This is because the isochrones for different ages, especially for young clusters, do not diverge sharply in the CMD from one another, but mostly extend to differing upper absolute magnitudes, so that isochrones of quite different ages can plausibly fit the same data. In contrast, for all ages the isochrones in the HRD have a fairly sharp bend at the high end of the main sequence which makes fitting to stellar data less ambiguous, as long as there are one or two stars that are at least about $50 \%$ of the way through their main sequence evolution.

Furthermore, the behaviour of the isochrones in the HRD is such that in most cases a reasonably clear assignment of the fitting uncertainty is easily possible, and this uncertainty is often remarkably small, of the order of $30 \%$ or less ( 0.1 dex).

The advantage of working in the HRD gradually diminishes with cluster age, as the isochrones in the CMD become more sharply bent, and is not great for cluster ages above perhaps 200 Myr.

We have re-determined the isochrone ages of seven nearby clusters. We conclude that our age determinations are precise (using a single set of isochrone computations, but including the effects of small differences in cluster compositions) to within less than about \pm 0.1 dex, or about $30 \%$, with a possible additional overall scale uncertainty (that does not affect the relative age determinations) due to uncertainties in stellar modelling physics, also of about 0.1 dex. These results represent a significant improvement in the determination of ages of most of the clusters studied.

A subsidiary aim of this work has been to test the current calibrations for obtaining $T_{\mathrm{e}}$ values of magnetic Ap stars from photometry. In general, the Ap stars in the clusters studied lie well within the spread of normal stars about the isochrones in the clusters studied. This result supports the adopted temperature calibration for these stars, although only at roughly the $10 \%$ level in the temperature range between about $9500 \mathrm{~K}$ and $15000 \mathrm{~K}$. The HRD positions we find for the Ap stars provide useful tests of cluster membership, and as discussed above are generally consistent with the claim that these stars are cluster members.

We find that our method of age determination facilitates the identification of blue stragglers in such favourable clusters, and we confirm the blue straggler nature of stars in IC 2602, NGC 2516, and NGC 6475, and show that several other proposed blue stragglers are not in fact cluster members.

Our overall conclusion is that where enough information is available to permit the secure determination of cluster membership, reddening, and chemical composition, and the stellar fundamental parameters $T_{\mathrm{e}}$ and $\log \left(L / L_{\odot}\right)$, studying clusters in the theoretical $\left(T_{\mathrm{e}}-\log \left(L / L_{\odot}\right)\right)$ Herzsprung-Russell diagram can provide valuable new insights, and securely determined stellar and cluster ages. Our methods can usefully be applied to obtain accurate ages for at least a dozen other nearby clusters (closer than, say, $400 \mathrm{pc}$ ) that contain several HIPPARCos stars and for which accurate parallaxes, mean proper motions, radial velocities, multi-colour photometry, and reddening are available (Robichon et al. 1999; Baumgardt et al. 2000).

Acknowledgements. We thank the two anonymous referees for very helpful comments on the paper. We have made extensive use of the SIMBAD astronomical database (http://simbad.u-strasbg.fr/simbad/sim-fid), the WEBDA database for open clusters (http://www.univie.ac.at/webda/), and the University of Lausanne photometric catalog (http://obswww. unige. $\mathrm{ch} / \mathrm{gcpd} / \mathrm{gcpd} . \mathrm{html}$ ). We are pleased to acknowledge funding provided by the Natural Sciences and Engineering Research Council of Canada.

\section{References}

Ahumada, J. A., \& Lapasset, E. 2007, A\&A, 463, 789

Alonso, A., Arribas, A., \& Martinez-Roger, G. 1999, A\&AS, 140, 261

Balona, L. A. 1994, MNRAS, 268, 119

Bagnulo, S., Landstreet, J. D., Lo Curto, G., Szeifert, T., \& Wade, G. A. 2003, A\&A, 403, 645

Bagnulo, S., Landstreet, J., Mason, E., et al. 2006, A\&A, 450, 777

Baumgardt, H., Dettbarn, C., \& Wielen, R. 2000, A\&AS, 146, 251

Basri, G., Martin, \& E. L. 1999, ApJ, 510, 266

Bertelli, G., Bressan, A., Chiosi, C., Fagotto, F., \& Nasi, E. 1994, A\&AS, 106 275

Boesgaard, A. M., Armengaud, E., \& King, J. R. 2003, ApJ, 582, 410

Bohlender, D. A., Landstreet, J. D., \& Thompson, I. B. 1993, A\&A, 269, 355

Bonatto, C., \& Bica, E. 2005, A\&A, 437, 483

Bressan, A., Fagotto, F., Bertelli, G., \& Chiosi, C. 1993, A\&AS, 100, 647

Bressan, A., Marigo, P., Girardi, L., et al. 2012, MNRAS, 427, 127

Brown, D. N., Shore, S. N., \& Sonneborn, G. 1985, AJ, 90, 1354

Buzzoni, A., Patelli, L., Bellazini, M., Fusi-Pecci, F., \& Oliva, E. 2010, MNRAS, 403, 1592

Carrier, F., Burki, G., \& Richard, C. 1999, A\&A, 341, 469

Cramer, N. 1984, A\&A, 132, 283

Crawford, D. L., \& Mandwewala, N. 1976, PASP, 88, 917

Dachs, J., \& Kabus, H. 1989, A\&AS, 78, 25

Dias, W. S., Lépine, J. R. D., \& Alessi, B. S. 2001, A\&A, 376, 441

ESA 1997, The HIPPARCos and Tycho Catalogues, ESA SP, 1200

Evans, N. R., Teays, T. J., Taylor, L. L., Lester, J. B., \& Hindsley, R. B. 1996, AJ, 111, 2099

Folsom, C. P., Wade, G. A., Bagnulo, S., \& Landstreet, J. D. 2007, MNRAS, 376,361

Gebran, M., Monier, R., \& Richard, O. 2008, A\&A, 479, 189

Girardi, L., Bressan, A., Bertelli, G., \& Chiosi, C. 2000, A\&AS, 141, 371

Golay, M. 1980, Vistas in Astron., 24, 141

Gonzales, J. F., \& Lapasset, E. 2000, AJ, 119, 2296

Hauck, B., \& North, P. 1993, A\&A, 269, 403

Hauck, B., \& Künzli, M. 1996, Baltic Astr., 5, 303

Hensberge, H., De Loore, C., Zuiderwijk, E. J., \& Hammerschlag-Hensberge, G. 1976, A\&A, 43, 383

Høg, E., Fabricius, C., Makarov, V. V., et al. 2000a, A\&A, 355, L27

Høg, E., Fabricius, C., Makarov, V. V., et al. 2000b, A\&A, 357, 367

Hubrig, S., Briquet, M., Morel, T., et al. 2008, A\&A, 488, 287

Hünsch, M., Randich, S. Hempel, M., Weidner, C., \& Schmitt, J. H. M. M. 2004, A\&A, 418, 539

Kharchenko, N. V., Piskunov, A. E., Röser, S., Schilbach, E., \& Scholz, R.-D. 2005, A\&A, 440, 403

Kudryavtsev, D. O., Romanyuk, I. I., Elkin, V. G., \& Paunzen, E. 2006, MNRAS, 372,1804

Künzli, M., North, P., Kurucz, R. L., \& Nicolet, B. 1997, A\&AS, 122, 51

Landstreet, J. D., Bagnulo, S., Andretta, V., et al. 2007, A\&A, 470, 685

Landstreet, J. D., Silaj, J., Andretta, V., et al. 2008, A\&A, 481, 465

Lloyd, C., Stickland, D., \& Walborn, N. R. 1995, PASP, 107, 1030

Loktin, A. V., Gerasimenko, T. P., \& Malysheva, L. K. 2001, Astron. Astrophys. Trans., 20, 607

Lucke, P. B. 1980, A\&A, 90, 350

Lyra, W., Moitinho, A., van der Bliek, N. S., \& Alves, J. 2006, A\&A, 453, 101 
Maiorca, E., Randich, S., Busso, M., Magrini, L., \& Palmerini, S. 2011, ApJ, 736,120

Makarov, V. V. 2006, ApJ, 131, 2967

Martin, E. L., Dahm, S., \& Pavlenko, Y. 2001, in Astrophysical Ages and Times Scales, eds. T. von Hippel, C. Simpson, \& N. Manset, ASP Conf. Ser., 245, 349

Masana, E., Jordi, C., \& Ribas, I. 2006, A\&A, 450, 735

Mermilliod, J.-C. 1981, A\&A, 97, 235

Mermilliod, J.-C., \& Paunzen, E. 2003, A\&A, 410, 511

Mermilliod, J.-C., Mermilliod, M., \& Hauck, B. 1997, A\&AS, 124, 349

Meynet, G., Mermilliod, J.-C., \& Maeder, A. 1993, A\&AS, 98, 477

Monroe, T. R., \& Pilachowski, C. A. 2010, AJ, 140, 2109

Monteiro, H., Dias, W. S., \& Caetano, T. C. 2010, A\&A, 516, A2

Napiwotzki, R., Schönberner, D., \& Wenske, V. 1993, A\&A, 268, 653

Naylor, T., \& Jeffries, R. D. 2006, MNRAS, 373, 1251

Pietrinferni, A., Cassisi, S., Salaris, M., \& Castelli, F. 2004, ApJ, 612, 168

Pinsonneault, M. H., Stauffer, J., Soderblom, D. R., King, J. R., \& Hanson R. B. 1998, ApJ, 504, 170

Platais, I., Kozhurina-Platais, V., Barnes, S., et al. 2001, AJ, 122, 1486
Randich, S. 2001, A\&A, 377, 512

Randich, S., Pallavicini, R., Meola, G., Stauffer, J. R., \& Balachandran, S. C. 2001, A\&A, 372, 862

Robichon, N., Arenou, F., Mermilliod, J.-C., \& Turon, C. 1999, A\&A, 345, 471 Sanders, W. L. 1971, A\&A, 14, 226

Sanner, J., \& Geffert, M. 2001, A\&A, 370, 87

Schaller, G., Schaerer, D., Meynet, G., \& Maeder, A. 1992, A\&AS, 96, 269

Semenko, E. A., Kudryavtsev, D. O., Ryabchikova, T. A., \& Romanyuk, I. I. 2008, Ap Bull, 63, 128

Sestito, P., Randich, S., Mermilliod, J.-C., \& Pallavicini, R. 2003, A\&A, 407, 289

Stauffer, J. R., Hartmann, L. W., Prosser, C. F., et al. 1997, ApJ, 479, 776

Stępień, K., \& Dominiczak, R. 1989, A\&A, 219, 197

Sung, H., Bessell, M. S., Lee, B.-W., \& Lee, S.-G. 2002, AJ, 123, 290

Tadross, A. L., Werner, P., Osman, A., \& Marie, M. 2002, New Astron., 7, 533

van Leeuwen, F. 2007, Hipparcos, the new reduction of the raw data (Dordrecht: Springer)

van Leeuwen, F. 2009, A\&A, 497, 209

Villanova, S., Carraro, G., \& Saviane, I. 2009, A\&A, 504, 845 
Table 3. Cluster members.

\begin{tabular}{|c|c|c|c|c|c|c|}
\hline HIP & Name & $M_{V}$ & $(B-V)_{0}$ & $T_{\mathrm{e}}(\mathrm{K})$ & $\log T_{\mathrm{e}}(\mathrm{K})$ & $\log L / L_{\odot}$ \\
\hline Cluster & IC 2602 & & & & & \\
\hline 50102 & HD 88980 & 2.778 & & 7312 & 3.864 & 0.756 \\
\hline 50612 & HD 89903 & 1.616 & & & & \\
\hline 51131 & HD 90731 & 1.496 & & & & \\
\hline 51203 & HD 90837 & 2.336 & & 8011 & 3.904 & 0.952 \\
\hline 51300 & HD 91043 & 3.256 & & & & \\
\hline 51576 & HD 91465 & -2.684 & -0.150 & 19291 & 4.285 & 3.717 \\
\hline 52059 & HD 92385, IC 260217 & 0.767 & -0.120 & 11100 & 4.045 & $1.783^{a}$ \\
\hline 52116 & HD 92467, IC 260218 & 1.016 & -0.010 & 10860 & 4.036 & 1.665 \\
\hline \multirow[t]{2}{*}{52132} & HD 92478, IC 260219 & 1.601 & 0.010 & 9925 & 3.997 & 1.361 \\
\hline & HD 92535, IC 260221 & 2.306 & 0.200 & 8016 & 3.904 & 0.964 \\
\hline 52160 & HD 92536, IC 260222 & 0.363 & -0.110 & 12114 & 4.083 & 2.024 \\
\hline 52221 & HD 92664, IC 260227 & -0.467 & -0.210 & 14200 & 4.152 & $2.512^{a}$ \\
\hline 52261 & HD 92715, IC 260228 & 0.846 & -0.060 & 10998 & 4.041 & 1.744 \\
\hline 52293 & HD 92783, IC 260229 & 0.751 & -0.090 & 11332 & 4.054 & 1.808 \\
\hline 52328 & HD 92837, IC 260231 & 1.207 & -0.040 & 10573 & 4.024 & 1.567 \\
\hline \multirow[t]{3}{*}{52370} & HD 92938, IC 260233 & -1.169 & -0.190 & 15397 & 4.187 & 2.877 \\
\hline & HD 92966, IC 260234 & 1.306 & -0.045 & 10550 & 4.023 & 1.526 \\
\hline & HD 92989, IC 260235 & 1.636 & 0.000 & 9951 & 3.998 & 1.349 \\
\hline \multirow[t]{2}{*}{52419} & HD 93030, IC 2602 37, $\theta$ Car & -3.224 & -0.270 & 30020 & 4.477 & $4.355^{c}$ \\
\hline & HD 93098, IC 260238 & 1.626 & 0.010 & 9727 & 3.988 & 1.337 \\
\hline \multirow[t]{2}{*}{52502} & HD 93194, IC 260241 & -1.176 & -0.185 & 16140 & 4.208 & 2.928 \\
\hline & HD 93517, IC 260247 & 1.886 & 0.055 & 9062 & 3.957 & 1.188 \\
\hline 52678 & HD 93540, IC 260248 & -0.641 & -0.140 & 14209 & 4.153 & 2.583 \\
\hline 52701 & HD 93549, IC 260249 & -0.733 & -0.130 & 14430 & 4.159 & 2.635 \\
\hline \multirow{2}{*}{52736} & HD 93607, IC 260251 & -1.116 & -0.190 & 16882 & 4.227 & 2.951 \\
\hline & HD 93648, IC 260252 & 1.896 & 0.075 & 8686 & 3.939 & 1.161 \\
\hline 52815 & HD 93738, IC 260254 & 0.516 & -0.020 & 11085 & 4.045 & 1.883 \\
\hline 52867 & HD 93874, IC 260258 & 2.234 & 0.130 & 8525 & 3.931 & 1.017 \\
\hline 53016 & HD 94174, IC 260263 & 1.796 & 0.060 & 9081 & 3.958 & 1.225 \\
\hline 53913 & HD 95786 & 1.526 & 0.030 & & & \\
\hline 54168 & HD 96287 & 1.256 & -0.040 & & & \\
\hline Cluster & NGC 2232 & & & & & \\
\hline \multirow[t]{3}{*}{30356} & HD 44720 & -0.432 & -0.140 & 13960 & 4.145 & 2.481 \\
\hline & HD 45051, NGC 223216 & 1.278 & 0.350 & & & \\
\hline & HD 45238, NGC 223212 & 0.758 & -0.060 & 11151 & 4.047 & 1.791 \\
\hline \multirow[t]{2}{*}{30660} & HD 45321, NGC 22322 & -1.486 & -0.170 & 16432 & 4.216 & 3.071 \\
\hline & HD 45399, NGC 223213 & 0.818 & -0.050 & 11526 & 4.062 & 1.796 \\
\hline \multirow[t]{2}{*}{30700} & HD 45418, NGC 22323 & -1.152 & -0.180 & & & \\
\hline & HD 45435, NGC 223218 & 1.528 & 0.030 & 8856 & 3.947 & 1.318 \\
\hline 30758 & HD 45516, NGC 22328 & 0.158 & -0.100 & 12752 & 4.106 & 2.155 \\
\hline 30761 & HD 45532, NGC 223210 & 0.428 & -0.080 & 12192 & 4.086 & 2.004 \\
\hline 30772 & HD 45546, NGC 22321 & -2.591 & -0.200 & 18790 & 4.274 & 3.653 \\
\hline \multirow[t]{2}{*}{30789} & HD 45583, NGC 22329 & 0.338 & -0.110 & 12700 & 4.104 & $2.079^{a}$ \\
\hline & HD 45627, NGC 223215 & 1.228 & 0.040 & 8935 & 3.951 & 1.443 \\
\hline \multirow[t]{4}{*}{31101} & HD 46165 & -0.192 & -0.090 & 13610 & 4.134 & 2.359 \\
\hline & HD 295100, NGC 223238 & 2.648 & 0.270 & 7292 & 3.863 & 0.807 \\
\hline & HD 295102, NGC 223214 & 1.028 & -0.070 & 11168 & 4.048 & 1.684 \\
\hline & NGC 223240 & 3.068 & 0.410 & & & \\
\hline \multirow[t]{4}{*}{ Cluster } & NGC 2451A & & & & & \\
\hline & CD-37 3859, NGC 2451A 70 & 3.677 & 0.420 & 6603 & 3.820 & 0.400 \\
\hline & CD-38 3604, NGC 2451A 24 & 2.871 & 0.240 & 7458 & 3.873 & 0.721 \\
\hline & HD 61621, NGC 2451A 27 & 1.740 & 0.040 & 9339 & 3.970 & 1.264 \\
\hline 37297 & HD 61831, NGC 2451 W201 & -1.509 & -0.200 & 16849 & 4.227 & 3.107 \\
\hline 37322 & HD 61878, NGC 2451A 60 & -0.641 & -0.130 & 13918 & 4.144 & $2.561^{b}$ \\
\hline 37450 & HD 62226, NGC 2451A 41 & -0.937 & -0.160 & 15115 & 4.179 & 2.765 \\
\hline \multirow[t]{3}{*}{37514} & HD 62376, NGC 2451A 43 & 0.179 & -0.100 & 13252 & 4.122 & $2.184^{b}$ \\
\hline & HD 62398, NGC 2451A 68 & 1.233 & -0.030 & 10304 & 4.013 & 1.536 \\
\hline & HD 62479, NGC 2451A 62 & 2.775 & 0.260 & 7394 & 3.869 & 0.758 \\
\hline 37557 & HD 62503, NGC 2451A 8 & 0.909 & -0.080 & & & \\
\hline \multirow[t]{2}{*}{37623} & HD 62578 & -0.751 & -0.140 & 14404 & 4.158 & 2.641 \\
\hline & HD 62642, NGC 2451A 87 & 1.246 & -0.020 & 9921 & 3.997 & 1.503 \\
\hline 37666 & HD 62712, NGC 2451A 54 & 0.078 & -0.170 & 14032 & 4.147 & 2.282 \\
\hline
\end{tabular}

Notes. ${ }^{(a)}$ Ap star; ${ }^{(b)}$ binary star; ${ }^{(c)}$ blue straggler. 
Table 3. continued.

\begin{tabular}{|c|c|c|c|c|c|c|}
\hline HIP & Name & $M_{V}$ & $(B-V)_{0}$ & $T_{\mathrm{e}}(\mathrm{K})$ & $\log T_{\mathrm{e}}(\mathrm{K})$ & $\log L / L_{\odot}$ \\
\hline \multirow{2}{*}{37697} & HD 62803, NGC 2451A 22 & 1.059 & -0.090 & 11050 & 4.043 & 1.663 \\
\hline & HD 62876, NGC 2451A 67 & 2.271 & 0.110 & 8515 & 3.930 & 1.002 \\
\hline \multirow[t]{3}{*}{37752} & HD 62893, NGC 2451A 78 & -0.459 & -0.130 & 13071 & 4.116 & 2.426 \\
\hline & HD 62961, NGC2451A 15 & 1.745 & 0.030 & 9428 & 3.974 & 1.268 \\
\hline & HD 62974, NGC 2451A 69 & 1.959 & 0.130 & 8286 & 3.918 & 1.115 \\
\hline 37838 & HD 63079, NGC 2451 A 88 & 0.649 & -0.080 & 11080 & 4.044 & 1.829 \\
\hline 37829 & HD 63080, NGC 2451A 2 & 0.849 & -0.040 & 11165 & 4.048 & 1.756 \\
\hline 37915 & HD 63215, NGC 2451A 79 & -0.471 & -0.120 & 14027 & 4.147 & $2.501^{b}$ \\
\hline \multirow[t]{2}{*}{37982} & HD 63401, NGC 2451A 4 & 0.001 & -0.160 & 13500 & 4.130 & $2.274^{a}$ \\
\hline & HD 63511, NGC 2451A 38 & 2.406 & 0.150 & 8348 & 3.922 & 0.939 \\
\hline 38268 & HD 64028 & 0.909 & -0.090 & 11765 & 4.071 & 1.778 \\
\hline Cluster & $\alpha$ Per & & & & & \\
\hline 14697 & Melotte 2056 & 4.381 & 0.720 & 5708 & 3.756 & 0.176 \\
\hline 14853 & BD+49 868, Melotte 20135 & 3.251 & 0.398 & 6723 & 3.828 & 0.568 \\
\hline 15911 & BD+46 745, Melotte 20632 & 3.257 & 0.386 & 6754 & 3.830 & 0.565 \\
\hline 15654 & BD+47 808, Melotte 20481 & 2.707 & 0.304 & 7100 & 3.851 & 0.782 \\
\hline 15898 & BD+47 816, Melotte 20621 & 3.401 & 0.399 & 6734 & 3.828 & 0.507 \\
\hline 15160 & BD+48 871, Melotte 20270 & 3.651 & 0.422 & 6671 & 3.824 & 0.409 \\
\hline 15363 & BD+49 897, Melotte 20365 & 3.441 & 0.407 & 6582 & 3.818 & 0.495 \\
\hline 16455 & BD+49 958, Melotte 20958 & 2.741 & 0.302 & 6996 & 3.845 & 0.769 \\
\hline 16625 & BD+49 967, Melotte 201050 & 3.021 & 0.309 & 6907 & 3.839 & 0.657 \\
\hline 16880 & BD+49 976, Melotte 201187 & 3.601 & 0.462 & 6238 & 3.795 & 0.446 \\
\hline 14949 & HD 19767, Melotte 20151 & 2.511 & 0.226 & 7559 & 3.878 & 0.867 \\
\hline 14980 & HD 19805, Melotte 20167 & 1.481 & 0.031 & 9443 & 3.975 & 1.375 \\
\hline 15040 & HD 19893, Melotte 20212 & 0.691 & -0.050 & 11291 & 4.053 & 1.829 \\
\hline 15259 & HD 20191, Melotte 20333 & 0.731 & -0.056 & 12051 & 4.081 & 1.872 \\
\hline 15388 & HD 20344, Melotte 20379 & 1.561 & & 8390 & 3.924 & 1.279 \\
\hline 15404 & HD 20365, Melotte 20383 & -1.309 & -0.151 & 16143 & 4.208 & 2.982 \\
\hline 15420 & HD 20391, Melotte 20386 & 1.481 & 0.026 & 9417 & 3.974 & 1.373 \\
\hline 15444 & HD 20418, Melotte 20401 & -1.439 & -0.170 & 15301 & 4.185 & 2.978 \\
\hline 15499 & HD 20475, Melotte 20421 & 2.771 & 0.363 & 6673 & 3.824 & 0.761 \\
\hline 15505 & HD 20487, Melotte 20423 & 1.181 & -0.017 & 9842 & 3.993 & 1.523 \\
\hline 15531 & HD 20510, Melotte 20441 & 0.591 & -0.040 & 11533 & 4.062 & 1.888 \\
\hline 15556 & HD 20537, Melotte 20450 & 0.840 & -0.060 & & & \\
\hline 15770 & HD 20809, Melotte 20557 & -1.159 & -0.166 & 15163 & 4.181 & 2.857 \\
\hline 15819 & HD 20863, Melotte 20581 & 0.531 & -0.075 & 11737 & 4.070 & 1.927 \\
\hline 15863 & HD 20902, Melotte 20605 & -4.629 & 0.391 & 6270 & 3.797 & 3.736 \\
\hline 15878 & HD 20931, Melotte 20612 & 1.411 & -0.002 & 10031 & 4.001 & 1.445 \\
\hline 15988 & HD 21071, Melotte 20675 & -0.399 & -0.169 & 14105 & 4.149 & 2.478 \\
\hline 16011 & HD 21091, Melotte 20692 & 1.036 & -0.056 & 10560 & 4.024 & 1.634 \\
\hline 16047 & HD 21117, Melotte 20703 & 1.171 & 0.000 & & & \\
\hline 16079 & HD 21181, Melotte 20735 & 0.376 & -0.106 & 11804 & 4.072 & 1.995 \\
\hline 16118 & HD 21238, Melotte 20747 & 0.466 & & 11227 & 4.050 & 1.914 \\
\hline 16147 & HD 21278, Melotte 20774 & -1.489 & -0.182 & 14970 & 4.175 & 2.975 \\
\hline 16137 & HD 21279, Melotte 20775 & 0.801 & -0.043 & 11867 & 4.074 & 1.829 \\
\hline 16210 & HD 21362, Melotte 20810 & -0.889 & -0.134 & 14073 & 4.148 & 2.672 \\
\hline 16318 & HD 21527, Melotte 20885 & 2.331 & 0.186 & 8036 & 3.905 & 0.955 \\
\hline 16340 & HD 21551, Melotte 20904 & -0.629 & -0.130 & 12577 & 4.100 & 2.456 \\
\hline 16403 & HD 21600, Melotte 20921 & 2.201 & 0.109 & 8518 & 3.930 & 1.030 \\
\hline 16426 & HD 21619, Melotte 20931 & 2.317 & 0.171 & 8163 & 3.912 & 0.966 \\
\hline 16430 & HD 21641, Melotte 20955 & 0.301 & -0.109 & 12074 & 4.082 & 2.046 \\
\hline 16470 & HD 21699, Melotte 20985 & -0.989 & -0.194 & 14400 & 4.158 & $2.735^{a}$ \\
\hline 16574 & HD 21855, Melotte 201056 & 1.731 & & 9102 & 3.959 & 1.252 \\
\hline 16782 & HD 22136, Melotte 201153 & 0.431 & -0.110 & 12770 & 4.106 & 2.047 \\
\hline 16826 & HD 22192, Melotte 201164 & -2.209 & -0.170 & 15654 & 4.195 & 3.310 \\
\hline 16966 & HD 22401, Melotte 201259 & 0.996 & -0.086 & 10859 & 4.036 & 1.673 \\
\hline 16995 & HD 22440, Melotte 201260 & 2.121 & & 8017 & 3.904 & 1.038 \\
\hline \multirow[t]{8}{*}{ Cluster } & NGC 2516 & & & & & \\
\hline & CPD-59 936, NGC 2516117 & 1.599 & -0.022 & 10344 & 4.015 & 1.393 \\
\hline & CPD-60 944, NGC 2516208 & 0.339 & -0.082 & 12600 & 4.100 & $2.071^{a}$ \\
\hline & CPD-60 945, NGC 25165 & 0.499 & -0.072 & 12543 & 4.098 & 2.002 \\
\hline & CPD-60 947, NGC 251613 & 0.051 & -0.152 & 12814 & 4.108 & 2.202 \\
\hline & CPD-60 948, NGC 25168 & 1.679 & 0.023 & 9039 & 3.956 & 1.269 \\
\hline & CPD-60 952, NGC 25169 & 1.268 & -0.022 & 9660 & 3.985 & 1.475 \\
\hline & CPD-60 961, NGC 25162 & 0.774 & -0.042 & 11304 & 4.053 & 1.797 \\
\hline
\end{tabular}


Table 3. continued.

\begin{tabular}{|c|c|c|c|c|c|c|}
\hline HIP & Name & $M_{V}$ & $(B-V)_{0}$ & $T_{\mathrm{e}}(\mathrm{K})$ & $\log T_{\mathrm{e}}(\mathrm{K})$ & $\log L / L_{\odot}$ \\
\hline \multirow{13}{*}{120403} & CPD-60 968, NGC 251641 & 0.972 & -0.062 & 8935 & 3.951 & 1.545 \\
\hline & CPD-60 969, NGC 251611 & 0.539 & -0.057 & 10579 & 4.024 & 1.835 \\
\hline & CPD-60 971, NGC 251658 & 1.655 & -0.032 & 10043 & 4.002 & 1.348 \\
\hline & CPD-60 975, NGC 25161 & 0.998 & -0.032 & 9697 & 3.987 & 1.586 \\
\hline & CPD-60 978, NGC 2516127 & 0.925 & -0.062 & 11800 & 4.072 & $1.775^{a}$ \\
\hline & CPD-60 979, NGC 251683 & 0.402 & -0.067 & 11905 & 4.076 & 1.992 \\
\hline & CPD-60 980, NGC 2516128 & -1.311 & 1.173 & 4490 & 3.652 & 2.607 \\
\hline & CPD-60 985, NGC 251637 & 0.199 & -0.122 & 11751 & 4.070 & 2.061 \\
\hline & CPD-60 989, NGC 251636 & 1.412 & -0.042 & 10188 & 4.008 & 1.456 \\
\hline & CPD-60 990, NGC 2516132 & 0.569 & -0.077 & 11885 & 4.075 & 1.924 \\
\hline & CPD-60 993, NGC 251648 & 0.779 & -0.112 & 12337 & 4.091 & 1.875 \\
\hline & CPD-60 1001, NGC 251633 & 1.453 & 0.078 & 9020 & 3.955 & 1.358 \\
\hline & CPD-60 1029, NGC 251665 & 1.859 & -0.022 & 9967 & 3.999 & 1.261 \\
\hline 38226 & HD 64320, NGC 2516225 & -1.301 & 1.128 & 4560 & 3.659 & 2.587 \\
\hline 38310 & HD 64507 & -0.787 & & 13526 & 4.131 & 2.591 \\
\hline 38433 & HD 64831 & -0.175 & & 12378 & 4.093 & 2.259 \\
\hline \multirow[t]{2}{*}{38536} & HD 65094, NGC 2516226 & -0.171 & -0.102 & 11705 & 4.068 & 2.206 \\
\hline & HD 65467, NGC 2516114 & 1.430 & -0.012 & 9765 & 3.990 & 1.418 \\
\hline 38739 & HD 65578, NGC 2516116 & 0.149 & -0.102 & 12218 & 4.087 & 2.117 \\
\hline 38759 & HD 65599, NGC 2516118 & 0.839 & -0.092 & 11206 & 4.049 & 1.763 \\
\hline 38783 & HD 65662, NGC 2516119 & -2.281 & 1.448 & 4060 & 3.608 & 3.162 \\
\hline \multirow[t]{5}{*}{38779} & HD 65663, NGC 2516120 & -1.329 & -0.102 & 12483 & 4.096 & $2.729^{b}$ \\
\hline & HD 65691, NGC 25166 & 0.909 & -0.067 & 11421 & 4.058 & 1.752 \\
\hline & HD 65712, NGC 2516230 & 1.359 & -0.092 & 9900 & 3.996 & $1.456^{a}$ \\
\hline & HD 65835, NGC 25163 & 1.453 & -0.037 & 10226 & 4.010 & 1.442 \\
\hline & HD 65869, NGC 251610 & -0.291 & -0.087 & 12169 & 4.085 & 2.290 \\
\hline \multirow[t]{2}{*}{120401} & HD 65896, NGC 251612 & 1.159 & -0.007 & 9816 & 3.992 & 1.530 \\
\hline & HD 65931, NGC 251639 & 0.769 & -0.022 & 11740 & 4.070 & 1.832 \\
\hline 120402 & HD 65949, NGC 251691 & 0.359 & -0.152 & 13240 & 4.122 & 2.111 \\
\hline 38906 & HD 65950, NGC 2516126 & -1.149 & -0.102 & 12842 & 4.109 & 2.685 \\
\hline \multirow[t]{2}{*}{120404} & HD 65987, NGC 251615 & -0.394 & -0.172 & 12600 & 4.100 & $2.364^{a}$ \\
\hline & HD 66137, NGC 251619 & -0.161 & -0.072 & 11445 & 4.059 & 2.181 \\
\hline \multirow[t]{4}{*}{38994} & HD 66194, NGC 2516134 & -2.192 & -0.212 & 20632 & 4.314 & $3.589^{c}$ \\
\hline & HD 66259, NGC 251620 & 0.373 & -0.092 & 11780 & 4.071 & 1.994 \\
\hline & HD 66295, NGC 251626 & 1.095 & -0.082 & 11100 & 4.045 & $1.652^{a}$ \\
\hline & HD 66318, NGC 251624 & 1.643 & -0.032 & 9100 & 3.959 & $1.287^{a}$ \\
\hline 39073 & HD 66341, NGC 2516136 & -1.695 & -0.167 & 13473 & 4.129 & 2.950 \\
\hline \multirow[t]{2}{*}{39070} & HD 66342, NGC 2516110 & -2.841 & 1.638 & 3820 & 3.582 & 3.572 \\
\hline & HD 66409, NGC 251623 & 0.389 & -0.102 & 12987 & 4.113 & 2.080 \\
\hline 39386 & HD 67170 & 0.099 & & 13175 & 4.120 & 2.210 \\
\hline 39438 & HD 67277 & 0.272 & & 13074 & 4.116 & 2.134 \\
\hline \multirow[t]{4}{*}{39879} & HD 68372 & -0.711 & & 13248 & 4.122 & 2.540 \\
\hline & NGC 2516209 & 0.759 & -0.042 & 12800 & 4.107 & $1.918^{a}$ \\
\hline & NGC 2516210 & -0.021 & -0.132 & 11300 & 4.053 & 2.114 \\
\hline & NGC 2516227 & 3.105 & 0.338 & 6672 & 3.824 & 0.627 \\
\hline Cluster & NGC 6475 & & & & & \\
\hline 87102 & HD 161575 & -0.423 & & 10500 & 4.021 & 2.206 \\
\hline 87134 & HD 161649 & 0.434 & & 13500 & 4.130 & $2.095^{c}$ \\
\hline 87240 & HD 161855 & -0.046 & 0.000 & 10250 & 4.011 & 2.033 \\
\hline \multirow[t]{6}{*}{87360} & HD 162144, NGC 6475131 & 0.275 & -0.050 & 10435 & 4.018 & 1.929 \\
\hline & HD 162223, NGC 64754 & 1.604 & & 7617 & 3.882 & $1.231^{b}$ \\
\hline & HD 162224, NGC 64756 & 1.774 & & 9083 & 3.958 & 1.234 \\
\hline & HD 162285, NGC 647512 & 1.364 & & 9264 & 3.967 & 1.410 \\
\hline & HD 162305, NGC 647514 & 0.494 & & 10600 & 4.025 & $1.817^{a}$ \\
\hline & HD 162349, NGC 647524 & 1.014 & & 9746 & 3.989 & 1.583 \\
\hline 87460 & HD 162374, NGC 647526 & -1.441 & -0.170 & 17004 & 4.231 & $3.089^{c}$ \\
\hline \multirow[t]{4}{*}{87472} & HD 162391, NGC 6475134 & -1.495 & 1.070 & 4800 & 3.681 & 2.621 \\
\hline & HD 162392, NGC 647528 & 1.664 & & 9177 & 3.963 & 1.284 \\
\hline & HD 162393, NGC 647529 & 0.724 & & 9761 & 3.989 & 1.700 \\
\hline & HD 162457, NGC 647534 & 0.794 & -0.020 & 9581 & 3.981 & 1.659 \\
\hline \multirow[t]{2}{*}{87516} & HD 162496 & -1.290 & 1.190 & 4500 & 3.653 & 2.606 \\
\hline & HD 162514, NGC 647540 & 1.214 & 0.190 & 7778 & 3.891 & $1.392^{b}$ \\
\hline 87529 & HD 162515, NGC 647542 & -0.784 & -0.040 & 10370 & 4.016 & 2.348 \\
\hline \multirow[t]{3}{*}{87560} & HD 162576, NGC 647555 & -0.353 & -0.020 & 10100 & 4.004 & $2.155^{a}$ \\
\hline & HD 162586, NGC 647556 & -1.236 & -0.110 & 12707 & 4.104 & $2.709^{c}$ \\
\hline & HD 162587, NGC 647558 & -1.606 & 1.020 & 4200 & 3.623 & 2.658 \\
\hline
\end{tabular}


Table 3. continued.

\begin{tabular}{|c|c|c|c|c|c|c|}
\hline HIP & Name & $M_{V}$ & $(B-V)_{0}$ & $T_{\mathrm{e}}(\mathrm{K})$ & $\log T_{\mathrm{e}}(\mathrm{K})$ & $\log L / L_{\odot}$ \\
\hline \multirow{4}{*}{87580} & HD 162588, NGC 647559 & -0.126 & & 10800 & 4.033 & $2.118^{a}$ \\
\hline & HD 162613 & 0.639 & & 10200 & 4.009 & 1.854 \\
\hline & HD 162678, NGC 6475141 & -1.076 & -0.060 & 9796 & 3.991 & 2.422 \\
\hline & HD 162679, NGC 647577 & -0.366 & -0.030 & 10638 & 4.027 & 2.201 \\
\hline 87616 & HD 162724, NGC 647586 & -1.346 & -0.070 & 10600 & 4.025 & 2.573 \\
\hline 87624 & HD 162725, NGC 647588 & -0.923 & -0.080 & 9600 & 3.982 & $2.347^{a}$ \\
\hline \multirow[t]{3}{*}{87656} & HD 162780, NGC 6475104 & -0.436 & -0.040 & 9902 & 3.996 & 2.174 \\
\hline & HD 162781, NGC 6475103 & 0.084 & 0.000 & 9221 & 3.965 & 1.919 \\
\hline & HD 162804, NGC 6475108 & -0.366 & -0.070 & 10648 & 4.027 & 2.202 \\
\hline 87671 & HD 162817, NGC 6475110 & -1.236 & -0.030 & 9500 & 3.978 & 2.442 \\
\hline 87686 & HD 162874 & 0.464 & & 10500 & 4.021 & 1.867 \\
\hline 87698 & HD 162888, NGC 6475121 & -0.401 & -0.040 & 9750 & 3.989 & 2.117 \\
\hline 87722 & HD 162926 & -1.286 & 0.020 & 9300 & 3.968 & 2.441 \\
\hline 87785 & HD 163109 & 0.616 & & 10300 & 4.013 & 1.741 \\
\hline 87798 & HD 163139 & -0.617 & & 10100 & 4.004 & 2.239 \\
\hline 87844 & HD 163251 & 0.348 & & 13095 & 4.117 & $2.105^{c}$ \\
\hline \multirow[t]{3}{*}{88247} & HD 164108 & 1.444 & & & & \\
\hline & HD 320764, NGC 647523 & 1.674 & & 8635 & 3.936 & 1.247 \\
\hline & HD 320765, NGC 647518 & 1.394 & & 8945 & 3.952 & 1.377 \\
\hline Cluster & Coma Ber & & & & & \\
\hline 59364 & HD 105805, Melotte 11110 & 1.310 & 0.114 & 8256 & 3.917 & 1.373 \\
\hline 59399 & HD 105863, Melotte 11112 & 4.849 & & 5944 & 3.774 & -0.033 \\
\hline 59527 & HD 106103, Melotte 11119 & 3.384 & 0.397 & 6653 & 3.823 & 0.516 \\
\hline 59833 & HD 106691, Melotte 11136 & 3.395 & 0.407 & 6668 & 3.824 & 0.511 \\
\hline 59957 & HD 106946, Melotte 11149 & 3.162 & 0.353 & 6899 & 3.839 & 0.601 \\
\hline 60025 & HD 107067, Melotte 11153 & 4.040 & 0.525 & 6222 & 3.794 & 0.271 \\
\hline 60066 & HD 107131, Melotte 11160 & 1.754 & 0.181 & 7895 & 3.897 & 1.180 \\
\hline 60063 & HD 107132, Melotte 11158 & 4.111 & 0.510 & 6195 & 3.792 & 0.244 \\
\hline 60087 & HD 107168, Melotte 11162 & 1.567 & 0.167 & 8026 & 3.904 & 1.260 \\
\hline 60123 & HD 107276, Melotte 11168 & 1.948 & 0.177 & 7876 & 3.896 & 1.102 \\
\hline 60206 & HD 107399, Melotte 11176 & 4.364 & 0.547 & 6056 & 3.782 & 0.152 \\
\hline 60266 & HD 107513, Melotte 11182 & 2.701 & 0.275 & 7340 & 3.866 & 0.787 \\
\hline 60293 & HD 107583, Melotte 11185 & 4.609 & 0.589 & 5998 & 3.778 & 0.058 \\
\hline 60304 & HD 107611, Melotte 11186 & 3.819 & 0.460 & 6408 & 3.807 & 0.350 \\
\hline 60347 & HD 107685, Melotte 11190 & 3.836 & 0.461 & 6440 & 3.809 & 0.342 \\
\hline 60351 & HD 107700, Melotte 11191 & 0.100 & 0.497 & 6169 & 3.790 & $1.850^{b}$ \\
\hline 60406 & HD 107793, Melotte 11197 & 4.410 & 0.540 & 6096 & 3.785 & 0.131 \\
\hline 60458 & HD 107877, Melotte 111101 & 3.673 & 0.443 & 6515 & 3.814 & 0.404 \\
\hline 60490 & HD 107935, Melotte 111104 & 2.006 & 0.237 & 7550 & 3.878 & 1.069 \\
\hline 60582 & HD 108102, Melotte 111111 & 3.429 & 0.518 & 6101 & 3.785 & $0.523^{b}$ \\
\hline 60611 & HD 108154, Melotte 111114 & 3.880 & 0.453 & 6348 & 3.803 & 0.328 \\
\hline 60649 & HD 108226, Melotte 111118 & 3.648 & 0.449 & 6511 & 3.814 & 0.415 \\
\hline 60697 & HD 108283, Melotte 111125 & 0.235 & 0.273 & 6841 & 3.835 & 1.772 \\
\hline 60746 & HD 108382, Melotte 111130 & 0.301 & 0.081 & 8340 & 3.921 & 1.781 \\
\hline 60797 & HD 108486, Melotte 111139 & 1.985 & 0.163 & 7923 & 3.899 & 1.089 \\
\hline 61071 & HD 108945, Melotte 111160 & 0.810 & 0.049 & 8800 & 3.944 & $1.602^{a}$ \\
\hline 61074 & HD 108976, Melotte 111162 & 3.864 & 0.475 & 6405 & 3.806 & 0.332 \\
\hline 61147 & HD 109069, Melotte 111398 & 2.860 & 0.330 & 6780 & 3.831 & 0.722 \\
\hline 61295 & HD 109307, Melotte 111183 & 1.574 & 0.108 & 8428 & 3.926 & 1.276 \\
\hline 61402 & HD 109483, Melotte 111192 & 5.270 & 0.570 & 6030 & 3.780 & -0.208 \\
\hline
\end{tabular}

